\title{
Weak convergence of obliquely reflected diffusions
}

\author{
Andrey Sarantsev \\ Department of Statistics and Applied Probability, UC Santa Barbara. E-mail: sarantsev@pstat.ucsb.edu
}

Received 8 September 2015; revised 2 May 2017; accepted 4 May 2017

\begin{abstract}
Burdzy and Chen (Electron. J. Probab. 3 (1998) 29-33) proved results on weak convergence of multidimensional normally reflected Brownian motions. We generalize their work by considering obliquely reflected diffusion processes. We require weak convergence of domains, which is stronger than convergence in Wijsman topology, but weaker than convergence in Hausdorff topology.
\end{abstract}

Résumé. Burdzy et Chen (Electron. J. Probab. 3 (1998) 29-33) ont montré des résultats portant sur la convergence faible des mouvements Browniens multidimensionnels avec réflexion normale. Nous généralisons leurs travaux dans le cas de processus de diffusion avec réflexion oblique. Notre résultat requiert la faible convergence des domaines. Notons que cette convergence est plus forte que la convergence dans la topologie de Wijsman, mais plus faible que celle de la topologie de Hausdorff.

MSC: Primary 60J60; secondary 60J55; 60J65; 60H10

Keywords: Reflected diffusions; Oblique reflection; Hausdorff convergence; Wijsman convergence; Weak convergence

\section{Introduction}

Consider a sequence of reflected diffusions $\left(Z_{n}\right)_{n \geq 0}$ : for every $n=0,1,2, \ldots$ let $Z_{n}=\left(Z_{n}(t), t \geq 0\right)$ be a reflected diffusion in $\bar{D}_{n}$, where $D_{n} \subseteq \mathbb{R}^{d}$ is an open connected subset (bounded or unbounded). When $Z_{n}(t) \in D_{n}$, this process is in the interior of its state space, it moves as a diffusion with drift vector field $g_{n}(\cdot)$ and covariance matrix field $A_{n}(\cdot)$. When $Z_{n}$ hits the boundary $\partial D_{n}$ at a point $z \in \partial D_{n}$, it is instantaneously reflected inside $D_{n}$, according to the direction $r_{n}(z)$. Here, $r_{n}: \partial D_{n} \rightarrow \mathbb{R}^{d}$ is a continuous vector field, defined on the boundary $\partial D_{n}$. In a more general setting, this boundary can have non-smooth parts (say, the origin for $D_{n}=(0, \infty)^{2}$ ); then the reflection field $r_{n}$ is defined everywhere on the boundary, except these non-smooth parts. If $r_{n}(z)$ is the inward unit normal vector to $\partial D_{n}$ at a point $z \in \partial D_{n}$, then this reflection is normal at this point $z$. Otherwise, it is oblique. We assume that the initial condition is $Z_{n}(0)=z_{n}$.

The main topic of this paper is: When do $Z_{n}$ weakly converge to $Z_{0}$ as elements of $C\left([0, T], \mathbb{R}^{d}\right)$ of continuous functions $[0, T] \rightarrow \mathbb{R}^{d}$ ? To establish this convergence, we need convergence of domains, drift vector fields and covariance matrix fields, reflection fields, and initial conditions:

$$
D_{n} \rightarrow D_{0}, \quad g_{n} \rightarrow g_{0}, \quad A_{n} \rightarrow A_{0}, \quad r_{n} \rightarrow r_{0}, \quad z_{n} \rightarrow z_{0} .
$$

But in which sense do we need to require this convergence? This article provides an answer to this question. The convergence of domains should be in what we call the weak sense, which is slightly stronger than in the Wijsman topology, see [1,21]. The convergence of functions poses certain problems, since they are defined on different domains. However, we find a way around this; we define what turns out to be a generalization of locally uniform convergence (and which, in fact, is locally uniform convergence if these functions, say $g_{n}$, are defined on the same domain). 
Convergence of reflected Brownian motions has been studied in [2] for the case of normal reflection and increasing sequence of domains $D_{n} \uparrow D_{0}$. In this article, we study this question in a more general setting: the reflection can be oblique, the concept of convergence $D_{n} \rightarrow D_{0}$ is more general than $D_{n} \uparrow D_{0}$, and we have general diffusion processes (with general drift and covariance fields instead of constant ones) instead of a Brownian motion.

However, in some sense our conditions are more restrictive: we require the boundary $\partial D_{n}$ to be smooth, except only a "small" subset; in the paper [2], it is only assumed that the boundary is continuous and the domain is bounded. In addition, we assume that the reflection fields $r_{n} \rightarrow r_{0}$ in a certain sense. In the paper [2], there is no additional assumption that reflection (in their case, normal) fields converge. Last but not least, in our paper the limiting process $Z_{0}$ should not hit non-smooth parts of the boundary. There are sufficient conditions for this to be true when the domain $D_{0}$ is a convex polyhedron, see for example [17,22]; see also a related paper [3]. An example of a reflected Brownian motion hitting or not hitting non-smooth parts of the boundary can be found in Proposition 3.1.

A related question is an invariance principle for a reflected Brownian motion in a convex polyhedron or, more generally, piecewise smooth domains. This has been studied in [11,24]. See also a recent paper [10] which uses similar techniques to prove well-posedness of a corresponding submartingale problem. We use similar techniques to our paper [19], which deals with penalty method for obliquely reflected diffusions. The difference is that the paper [19] approximated an obliquely reflected diffusion by a solution of an SDE without reflection, but with an appropriately chosen drift vector field. The current paper approximates on obliquely reflected diffusion by another obliquely reflected diffusion.

\subsection{Organization of the paper}

Section 2 contains definitions and the main result (Theorem 2.7). In Section 3, we apply these results to reflected Brownian motion in the orthant and in other convex polyhedral domains. Section 4 is devoted to the proof of Theorem 2.7. Section 5 contains results for the case when $D_{n} \rightarrow \mathbb{R}^{d}$, that is, the limiting process $Z_{0}$ is actually a non-reflected diffusion. The Appendix contains some technical lemmata.

\subsection{Notation}

For a vector or a matrix $a$, the symbol $a^{\prime}$ denotes the transpose of $a$. Denote the weak convergence by $\Rightarrow$. Let $C\left([0, T], \mathbb{R}^{d}\right)$ be the space of all continuous functions $[0, T] \rightarrow \mathbb{R}^{d}$, with the max-norm. For $d=1$, we simply write $C[0, T]$. For two vectors $a=\left(a_{1}, \ldots, a_{d}\right)^{\prime}$ and $b=\left(b_{1}, \ldots, b_{d}\right)^{\prime}$ in $\mathbb{R}^{d}$, we denote their dot product by $a \cdot b=$ $a_{1} b_{1}+\cdots+a_{d} b_{d}$. The Euclidean norm of $a$ is given by $\|a\|=\left[a_{1}^{2}+\cdots+a_{d}^{2}\right]^{1 / 2}$. For $x=\left(x_{1}, \ldots, x_{d}\right)^{\prime} \in \mathbb{R}^{d}$ and $y=\left(y_{1}, \ldots, y_{d}\right)^{\prime} \in \mathbb{R}^{d}$, we write $x \geq y$ if $x_{i} \geq y_{i}$ for $i=1, \ldots, d$ and $x>y$ if $x_{i}>y_{i}$ for $i=1, \ldots, d$; similarly for $x \leq y$ and $x<y$. For $x \in \mathbb{R}^{d}, \varepsilon>0$, let $U(x, \varepsilon):=\left\{y \in \mathbb{R}^{d} \mid\|x-y\|<\varepsilon\right\}$ be the $\varepsilon$-neighborhood of $x$. For a point $x \in \mathbb{R}^{d}$ and a set $E \subseteq \mathbb{R}^{d}$, denote the distance from $x$ to $E$ by $\operatorname{dist}(x, E)$. For a set $E \subseteq \mathbb{R}^{d}$ and $r>0$, denote $U_{r}(E)=\left\{x \in \mathbb{R}^{d} \mid \operatorname{dist}(x, E)<r\right\}$. For two sets $E, F \subseteq \mathbb{R}^{d}$, denote the distance from $E$ to $F$ by $\operatorname{dist}(E, F)$. For a subset $E \subseteq \mathbb{R}^{d}$, we denote the set of its interior points by int $E$, and the complement $\mathbb{R}^{d} \backslash E$ by $E^{c}$. We denote its closure by $\bar{E}$. We write $f \in C^{r}$ for $r$ times continuously differentiable function $f$, defined on some subset of $\mathbb{R}^{d}$. We also say that a subset $E$ of $\mathbb{R}^{d}$ is $C^{r}$ if $E$ is an $r$ times continuously differentiable hypersurface in $\mathbb{R}^{d}$. The symbol $\operatorname{mes}(E)$ denotes the Lebesgue measure of a set $E$ in $\mathbb{R}$ or $\mathbb{R}^{d}$, depending on the context. The set of all $d \times d$ positive definite symmetric matrices is denoted by $P_{d}$. Define the modulus of continuity for a function $f: \mathbb{R}_{+} \rightarrow \mathbb{R}^{d}$ : for $T>0$ and $\delta>0$,

$$
\omega(f,[0, T], \delta):=\sup _{\substack{t, s \in[0, T] \\|t-s| \leq \delta}}\|f(t)-f(s)\| .
$$

\section{Definitions and the main result}

\subsection{Definition of a reflected diffusion}

Fix $d \geq 1$, the dimension. Consider a domain (open connected subset) $D \subseteq \mathbb{R}^{d}$. Take a function $g: \bar{D} \rightarrow \mathbb{R}^{d}$ and a matrix-valued function $A: \bar{D} \rightarrow P_{d}$. Let $\sigma(x):=A^{1 / 2}(x)$ be the positive definite matrix square root of $A(x)$. Assume 
that the boundary $\partial D$ is $C^{2}$ everywhere, except a closed subset $\mathcal{V} \subseteq \partial D$; that is, $\partial D \backslash \mathcal{V}$ is $C^{2}$. The set $\mathcal{V}$ is called an exceptional set, or non-smooth parts of the boundary $\partial D$. For example, if $D=\operatorname{int} S$, where $S:=\mathbb{R}_{+}^{d}$ is an interior of the positive $d$-dimensional orthant, then the boundary $\partial D=\partial S$ consists of $d$ faces: $S_{i}:=\left\{x \in S \mid x_{i}=0\right\}$, and $\mathcal{V}:=\bigcup_{1 \leq i<j \leq d}\left(D_{i} \cap D_{j}\right)$. If $D$ is smooth, or, more precisely, the whole boundary $\partial D$ is $C^{2}$, then we let $\mathcal{V}:=\varnothing$.

Denote for $x \in \partial D \backslash \mathcal{V}$ the inward unit normal vector by $\mathfrak{n}(x)$. Take a vector field $r: \partial D \backslash \mathcal{V} \rightarrow \mathbb{R}^{d}$ such that $r(x) \cdot \mathfrak{n}(x)>0$. (Without loss of generality, we can assume $r(x) \cdot \mathfrak{n}(x)=1$; a very short proof of this fact is given in [19].) The function $r$ is called a reflection field. We note that the set $\mathcal{V}$ includes, but is not limited to, the parts of the boundary $\partial D$ where it is not $C^{2}$. It also might include points of the boundary where $\partial D$ is smooth, but the reflection field $r$ is undefined. Slightly abusing the notation, we call the collection of all these points non-smooth parts of the boundary.

We would like to define a reflected diffusion $Z=(Z(t), t \geq 0)$ in $\bar{D}$ with drift coefficient $g$, covariance matrix $A$, and reflection field $r$. This is a process that:

(i) behaves as a solution of an SDE with drift coefficient $g$ and covariance matrix $A$, so long as it stays inside $D$;

(ii) when it hits the boundary $\partial D$ at a point $x \in \partial D \backslash \mathcal{V}$, it reflects according to the reflection vector $r(x)$; if $r(x)=$ $\mathfrak{n}(x)$, this reflection is called normal, and otherwise it is called oblique.

Definition 1. Take $d$ i.i.d. standard Brownian motions $W_{1}, \ldots, W_{d}$ and let $W=\left(W_{1}, \ldots, W_{d}\right)^{\prime}$. A continuous adapted process $Z=(Z(t), t \geq 0)$ with values in $\bar{D}$ is called a reflected diffusion in $\bar{D}$, stopped after hitting $\mathcal{V}$ with drift vector field $g$, covariance matrix field $A$, and reflection field $r$, starting from $Z(0)=z_{0}$, if there exists a real-valued continuous adapted nondecreasing process $l=(l(t), t \geq 0)$ with $l(0)=0$, such that $l$ can increase only when $Z \in \partial D$, and

$$
Z(t)=z_{0}+\int_{0}^{t \wedge \tau \mathcal{V}} g(Z(s)) \mathrm{d} s+\int_{0}^{t \wedge \tau \mathcal{V}} \sigma(Z(s)) \mathrm{d} W(s)+\int_{0}^{t \wedge \tau \mathcal{V}} r(Z(s)) \mathrm{d} l(s), \quad t \geq 0,
$$

where $\tau \mathcal{V}:=\min \{t \geq 0 \mid Z(t) \in \mathcal{V}\}$, and $\sigma(x):=A^{1 / 2}(x)$ is the positive definite symmetric square root of the matrix $A(x)$, for every $x \in \bar{D}$. The process $L(t):=\int_{0}^{t \wedge \tau \mathcal{V}} r(Z(s)) \mathrm{d} l(s), t \geq 0$, is called the reflection term. We say this reflected diffusion avoids non-smooth parts of the boundary if $\tau_{\mathcal{V}}=\infty$ a.s.

We can write (1) in the differential form:

$$
\mathrm{d} Z(t)=g(Z(t)) \mathrm{d} t+\sigma(Z(t)) \mathrm{d} W(t)+r(Z(t)) \mathrm{d} l(t), \quad t<\tau \mathcal{V} .
$$

The property that $l$ can increase only when $Z \in \partial D$ can be written formally as

$$
\int_{0}^{\infty} 1(Z(t) \in D) \mathrm{d} l(t)=0 .
$$

There are several conditions for weak or strong existence and uniqueness of this diffusion, discussed in the articles mentioned in the Introduction. In this article, we simply assume that it exists in the weak sense, is unique in law, and does not hit non-smooth parts of the boundary. More precisely, let us state the following assumptions.

Assumption 1. The exceptional set $\mathcal{V}$ is "small enough"; namely, for every $x \in \mathbb{R}^{d}$ we have:

$$
\operatorname{dist}(x, \partial D)=\operatorname{dist}(x, \partial D \backslash \mathcal{V}) .
$$

For example, this is true for an orthant $D=(0, \infty)^{d}$, or a convex polyhedron $D$ (see Section 3).

Assumption 2. The reflection field $r: \partial D \backslash \mathcal{V} \rightarrow \mathbb{R}^{d}$ is continuous on $\partial D \backslash \mathcal{V}$. Moreover, as mentioned above, $r(z) \cdot \mathfrak{n}(z)=1$ for $z \in \partial D \backslash \mathcal{V}$.

Assumption 3. The reflected diffusion from Definition 1 with parameters $g, A, r$, starting from $z_{0}$, exists and is unique in the weak sense. 
A particular case of a reflected diffusion is reflected Brownian motion, when the drift coefficient $g(x)$ and the covariance matrix $A(x)$ do not depend on $x: g(x) \equiv g$, and $A(x) \equiv A$. An example of a reflected Brownian motion hitting or not hitting non-smooth parts of the boundary is given in Section 3, Proposition 3.1.

\subsection{Weak convergence of domains}

For each $n=0,1,2, \ldots$, define the function $\varphi_{n}: \mathbb{R}^{d} \rightarrow \mathbb{R}$ to be the signed distance to $\partial D_{n}$ :

$$
\varphi_{n}(x):= \begin{cases}\operatorname{dist}\left(x, \partial D_{n}\right), & x \in D_{n} ; \\ 0, & x \in \partial D_{n} ; \\ -\operatorname{dist}\left(x, \partial D_{n}\right), & x \in \mathbb{R}^{d} \backslash \overline{D_{n}} .\end{cases}
$$

Definition 2. We say that the sequence of domains $\left(D_{n}\right)_{n \geq 1}$ converges weakly to the domain $D_{0}$ in $\mathbb{R}^{d}$, and write $D_{n} \Rightarrow D_{0}$, if $\varphi_{n}(x) \rightarrow \varphi_{0}(x)$ for every $x \in \mathbb{R}^{d}$.

There are other well-known concepts of set convergence in $\mathbb{R}^{d}$.

Definition 3. Take subsets $E_{n} \subseteq \mathbb{R}^{d}, n=0,1,2, \ldots$ We say that $E_{n} \rightarrow E_{0}$ in Wijsman topology if dist $\left(x, E_{n}\right) \rightarrow$ $\operatorname{dist}\left(x, E_{0}\right)$ for all $x \in \mathbb{R}^{d}$. If this convergence is uniform for $x \in \mathbb{R}^{d}$, then $E_{n} \rightarrow E_{0}$ in Hausdorff topology. An equivalent definition of Hausdorff convergence is through Hausdorff distance, which is defined for $A, B \subseteq \mathbb{R}^{d}$ as follows:

$$
d_{H}(A, B)=\inf \left\{\varepsilon>0 \mid A \subseteq U_{\varepsilon}(B) \text { and } B \subseteq U_{\varepsilon}(A)\right\} .
$$

For Wijsman convergence, we can substitute $E_{n}$ by their closures, because

$$
\operatorname{dist}\left(x, E_{n}\right) \equiv \operatorname{dist}\left(x, \bar{E}_{n}\right) .
$$

There are equivalent definitions of Hausdorff convergence, distance and topology. We refer the reader to the book [13]. For Wijsman convergence, see the articles $[1,21]$. In a sense, both Wijsman convergence and weak convergence are "local" analogues of Hausdorff convergence, just as locally uniform convergence of functions with respect to uniform convergence. Let us state a few elementary properties of Wijsman and weak convergence, with the proofs postponed until Appendix.

Lemma 2.1. Suppose $E_{n} \rightarrow E_{0}$ in Wijsman topology. Then:

(i) $\operatorname{dist}\left(x, E_{n}\right) \rightarrow \operatorname{dist}\left(x, E_{0}\right)$ uniformly on every compact subset $\mathcal{K} \subseteq \mathbb{R}^{d}$;

(ii) if $x_{n} \in E_{n}$ and $x_{n} \rightarrow x_{0}$, then $x_{0} \in \bar{E}_{0}$.

Lemma 2.2. The following statements for domains $D_{n}, n=0,1,2, \ldots$ are equivalent:

(i) $D_{n} \Rightarrow D_{0}$;

(ii) $D_{n} \rightarrow D_{0}$ and $D_{n}^{c} \rightarrow D_{0}^{c}$ in Wijsman topology;

(iii) $\varphi_{n}(x) \rightarrow \varphi_{0}(x)$ uniformly on every compact subset $\mathcal{K} \subseteq \mathbb{R}^{d}$;

(iv) for every compact subset $\mathcal{K} \subseteq \mathbb{R}^{d}$ and a sequence $\left(\varepsilon_{n}\right)_{n \geq 1}$ with $\varepsilon_{n} \rightarrow 0$, we have:

$$
\max _{\substack{x_{n}, x_{0} \in \mathcal{K} \\\left\|x_{n}-x_{0}\right\| \leq \varepsilon_{n}}}\left|\varphi_{n}\left(x_{n}\right)-\varphi_{0}\left(x_{0}\right)\right| \rightarrow 0 \quad \text { as } n \rightarrow \infty ;
$$

(v) for every $T>0$ and every sequence $\left(f_{n}\right)_{n \geq 1}$ of functions $f_{n}:[0, T] \rightarrow \mathbb{R}^{d}$ which converges uniformly on $[0, T]$ to a continuous function $f_{0}:[0, T] \rightarrow \mathbb{R}^{d}$, we have: $\varphi_{n}\left(f_{n}(\cdot)\right) \rightarrow \varphi_{0}\left(f_{0}(\cdot)\right)$ uniformly on $[0, T]$; 
(vi) $\partial D_{n} \rightarrow \partial D_{0}$ in Wijsman topology, and, in addition,

$$
D_{0} \subseteq \underset{n \rightarrow \infty}{\lim _{n \rightarrow \infty}} D_{n} \text { and } \varlimsup_{n \rightarrow \infty} \bar{D}_{n} \subseteq \bar{D}_{0} ;
$$

(vii) if $x_{n_{k}} \in \partial D_{n_{k}}$ and $x_{n_{k}} \rightarrow x_{0}$ for some subsequence $\left(n_{k}\right)_{k \geq 1}$, then $x_{0} \in \partial D_{0}$, and (2) holds.

Corollary 2.3. Assume $D_{n} \Rightarrow D_{0}$.

(i) Take a sequence $\left(x_{k}\right)_{k \geq 1}$. If $x_{k} \in \overline{D_{n_{k}}}$ for some subsequence $\left(n_{k}\right)_{k \geq 1}$, and $x_{k} \rightarrow x_{0}$, then $x_{0} \in \overline{D_{0}}$. If $x_{k} \in D_{n_{k}}^{c}$, and $x_{k} \rightarrow x_{0}$, then $x_{0} \in D_{0}^{c}$. If $x_{k} \rightarrow x_{0}$, and $x_{k} \in \partial D_{n_{k}}$, then $x_{0} \in \partial D_{0}$.

(ii) For every compact subset $\mathcal{K} \subseteq D_{0}$, there exists $n_{0}$ such that for $n>n_{0}$, we have: $\mathcal{K} \subseteq D_{n}$. For every compact subset $\mathcal{K} \subseteq \bar{D}_{0}^{c}$, there exists $n_{0}$ such that for $n>n_{0}$, we have: $\mathcal{K} \subseteq \bar{D}_{n}^{c}$.

When $\left(D_{n}\right)_{n \geq 1}$ is a monotone sequence, this concept of convergence can be simplified.

Lemma 2.4. If $D_{n} \uparrow D_{0}$ or $\bar{D}_{n} \downarrow \bar{D}_{0}$, then $D_{n} \Rightarrow D_{0}$.

The following lemma provides comparison of convergence modes.

\section{Lemma 2.5.}

(i) Weak convergence $D_{n} \Rightarrow D_{0}$ is stronger than Wijsman convergence.

(ii) Weak convergence $D_{n} \Rightarrow D_{0}$ is weaker than Hausdorff convergence.

(iii) $D_{n} \rightarrow D_{0}$ in Hausdorff topology if and only if $\varphi_{n}(x) \rightarrow \varphi_{0}(x)$ uniformly on the whole $\mathbb{R}^{d}$.

Example 1. Fix $d \geq 2$, the dimension. Let $e_{1}=(1,0, \ldots, 0)^{\prime} \in \mathbb{R}^{d}$. Consider a sequence $D_{n}:=U\left(n e_{1}, n\right)$ of open balls of radius $n$ centered at $n e_{1}$. This is an increasing sequence: $D_{n} \subseteq D_{n+1}$. It is easy to see that $D_{n} \uparrow D_{0}=\{x \in$ $\left.\mathbb{R}^{d} \mid x_{1}>0\right\}$. By Lemma 2.4, $D_{n} \Rightarrow D_{0}$.

Example 2. Take a sequence $D_{n}=U\left(x_{n}, a_{n}\right)$ of open discs in $\mathbb{R}^{d}$. Then $D_{n} \Rightarrow D_{0}$ if and only if $x_{n} \rightarrow x_{0}$ and $a_{n} \rightarrow a_{0}$. Indeed, $\varphi_{n}(x) \equiv a_{n}-\left\|x-x_{n}\right\|$, so the "if" part is obvious. Let us show the "only if" part. Assume $D_{n} \Rightarrow D_{0}$. Take an arbitrarily small $\varepsilon>0$. Then by Corollary 2.3 , for $\mathcal{K}=\overline{U\left(x_{0}, a_{0}-\varepsilon\right)} \subseteq D_{0}$, there exists $n_{0}$ such that for $n>n_{0}$ we have: $\mathcal{K} \subseteq D_{n}=U\left(x_{n}, a_{n}\right)$. But if $\overline{U\left(y_{1}, a_{1}\right)} \subseteq U\left(y_{2}, a_{2}\right)$, then $a_{1}<a_{2}$ and $\left\|y_{1}-y_{2}\right\| \leq a_{2}-a_{1}$. Therefore,

$$
a_{0}-\varepsilon<a_{n} \quad \text { and } \quad\left\|x_{n}-x_{0}\right\| \leq a_{n}-a_{0}+\varepsilon \quad \text { for } n>n_{0} .
$$

We can take arbitrarily small $\varepsilon>0$. From the first comparison in (3),

$$
\varliminf_{n \rightarrow \infty} a_{n} \geq a_{0}
$$

Similarly, taking $\mathcal{K}=U(0, N) \backslash U\left(x_{0}, a_{0}+\varepsilon\right)$ for large $N$ and small $\varepsilon>0$, we conclude: $\mathcal{K} \subseteq \bar{D}_{0}^{c}$, and so $\mathcal{K} \subseteq \bar{D}_{n}^{c}$ for large enough $n$. Therefore, $a_{n} \leq a_{0}+\varepsilon$. This leads to the conclusion that

$$
\varlimsup_{n \rightarrow \infty} a_{n} \leq a_{0} .
$$

Combining (4) and (5), we get: $a_{n} \rightarrow a_{0}$. Now, from the second comparison in (3) we have: because $a_{n} \rightarrow a_{0}$ and $\varepsilon>0$ is arbitrarily small, $x_{n} \rightarrow x_{0}$.

Example 3. Take a sequence $\left(f_{n}\right)_{n \geq 1}$ of smooth functions $\mathbb{R}_{+}^{d-1} \rightarrow \mathbb{R}$ such that $f_{n} \rightarrow 0$ locally uniformly and $f_{n}(0)=$ 0 . For $i=1, \ldots, d$ and $x=\left(x_{1}, \ldots, x_{d}\right)^{\prime}$, we let

$$
\hat{x}_{i}=\left(x_{1}, \ldots, x_{i-1}, x_{i+1}, \ldots, x_{d}\right)^{\prime} \in \mathbb{R}^{d-1} .
$$


Now, define the following sequence of domains:

$$
D_{n}=\left\{x \in \mathbb{R}^{d} \mid x_{i}>f_{n}\left(\hat{x}_{i}\right), i=1, \ldots, d\right\} .
$$

Then $D_{n} \Rightarrow D_{0}=(0, \infty)^{d}$. The proof is similar to that of Theorem 3.2(i), (ii) below.

\subsection{Main result}

Consider a sequence $\left(D_{n}\right)_{n \geq 1}$ of domains in $\mathbb{R}^{d}$. Let $\mathcal{V}_{n}$ be non-smooth parts of the boundary for $D_{n}$. For each $n=0,1,2, \ldots$ take a reflected diffusion $Z_{n}$ in $\bar{D}_{n}$ with drift vector $g_{n}$, covariance matrix $A_{n}$, and reflection field $r_{n}$, starting from $z_{n}=Z_{n}(0)$. Suppose that for every $n=0,1,2, \ldots$, this reflected diffusion $Z_{n}$ satisfies Assumptions 1-3.

The main question of this paper is:

Under what assumptions on $g_{n}, A_{n}, r_{n}, z_{n}, D_{n}$, do we have:

$$
Z_{n} \Rightarrow Z_{0} \quad \text { weakly in } C\left([0, T], \mathbb{R}^{d}\right) \text { for every } T>0 \text { ? }
$$

First, we need the domains $D_{n}$ to converge to $D_{0}$ in some sense. We already defined an appropriate concept of weak convergence earlier. We also need to have

$$
g_{n} \rightarrow g_{0}, \quad A_{n} \rightarrow A_{0}, \quad r_{n} \rightarrow r_{0}
$$

uniformly in some sense. But these functions are defined on different subsets of $\mathbb{R}^{d}$. A natural way to define convergence is as follows.

Definition 4. Take functions $f_{n}: E_{n} \rightarrow \mathbb{R}^{p}, n=0,1,2, \ldots$ where $E_{n} \subseteq \mathbb{R}^{d}$, and $p \geq 1$ is some dimension. We say that $f_{n} \rightarrow f_{0}$ locally uniformly, and write $f_{n} \Rightarrow f_{0}$, if one of these two equivalent statements is true:

(i) for every subsequence $\left(n_{k}\right)_{k \geq 1}$ and any sequence $\left(z_{n_{k}}\right)_{k \geq 1}$ such that $z_{n_{k}} \in E_{n_{k}}$ and $z_{n_{k}} \rightarrow z_{0} \in E_{0}$ we have: $f_{n_{k}}\left(z_{n_{k}}\right) \rightarrow f_{0}\left(z_{0}\right)$

(ii) for every $T>0$, and for every subsequence $\left(n_{k}\right)_{k \geq 1}$ and any sequence $\left(x_{n_{k}}\right)_{k \geq 1}$ of continuous functions [0,T] $\rightarrow$ $\mathbb{R}^{d}$ such that $x_{n}(t) \in E_{n}$ for all $n=0,1, \ldots$ we have:

$$
\begin{aligned}
& \text { if } x_{n_{k}}(t) \rightarrow x_{0}(t) \quad \text { uniformly on }[0, T], \\
& \text { then } f_{n_{k}}\left(x_{n_{k}}(t)\right) \rightarrow f_{0}\left(x_{0}(t)\right) \text { uniformly on }[0, T] .
\end{aligned}
$$

Lemma 2.6. These two definitions (i) and (ii) of locally uniform convergence are indeed equivalent, if $f_{0}$ is continuous on $E_{0}$.

The proof of Lemma 2.6 is postponed until Appendix.

Remark 1. In the case $E_{n}=E_{0}$, if the function $f_{0}$ is continuous, then $f_{n} \Rightarrow f_{0}$ is equivalent to the locally uniform convergence on $E_{0}$ in the usual sense (that is, uniform convergence on $E_{0} \cap \mathcal{K}$ for every compact set $\mathcal{K} \subseteq \mathbb{R}^{d}$ ).

Remark 2. Note that $A_{n} \Rightarrow A_{0}$ if and only if $\sigma_{n}(x):=A_{n}^{1 / 2}(x) \Rightarrow \sigma_{0}(x):=A_{0}^{1 / 2}(x)$. The "if" part follows from the obvious fact that the operation of taking the square of a matrix is continuous. The "only if" part follows from the fact that the operation of taking a symmetric positive definite square root of a symmetric positive definite matrix is also continuous, see for example [7].

Now comes the main result of this paper.

Theorem 2.7. Take $Z_{n}$ for $n=0,1,2, \ldots$ as described above. Assume each $Z_{n}, n=0,1,2, \ldots$ satisfies Assumptions 1-3. Suppose that $g_{0}, A_{0}, r_{0}$ are locally bounded, and $Z_{0}$ does not hit non-smooth parts of the boundary. 
Assume that

$$
D_{n} \Rightarrow D_{0}, \quad g_{n} \Rightarrow g_{0}, \quad A_{n} \Rightarrow A_{0}, \quad r_{n} \Rightarrow r_{0}, \quad z_{n} \rightarrow z_{0} .
$$

Also, assume that at least one of the following conditions (a) or (b) holds true:

(a) for all $n \geq n_{0}$, the process $Z_{n}$ does not hit non-smooth parts $\mathcal{V}_{n}$ of the boundary $\partial D_{n}$;

(b) for every compact set $\mathcal{K} \subseteq \mathbb{R}^{d}$, we have:

$$
\lim _{n \rightarrow \infty} \max _{x \in \mathcal{V}_{n} \cap \mathcal{K}} \operatorname{dist}\left(x, \mathcal{V}_{0}\right)=0 .
$$

Then $Z_{n} \Rightarrow Z_{0}$ weakly in $C\left([0, T], \mathbb{R}^{d}\right)$ for every $T>0$.

The following is a necessary and sufficient condition for (6).

Lemma 2.8. Condition (b) from Theorem 2.7 holds if and only if for every sequence $\left(x_{n_{k}}\right)_{k \geq 1}$ with $x_{n_{k}} \in \mathcal{V}_{n_{k}}$ and $x_{n_{k}} \rightarrow x_{0}$ we have: $x_{0} \in \mathcal{V}_{0}$. In particular, we can apply Lemma 2.1(i) and conclude: condition (b) holds if $\mathcal{V}_{n} \rightarrow \mathcal{V}_{0}$ in Wijsman topology.

If all domains $D_{0}, D_{1}, D_{2}, \ldots$ are the same, then we can restate this main result as follows.

Corollary 2.9. Assume $D_{n}=D$ for $n=0,1,2, \ldots$, where $D$ has non-smooth parts of the boundary $\mathcal{V}$. Suppose $r_{n} \rightarrow r_{0}$ locally uniformly on $\partial D \backslash \mathcal{V}$, and $g_{n} \rightarrow g_{0}, \sigma_{n} \rightarrow \sigma_{0}$ locally uniformly on $\bar{D} \backslash \mathcal{V}$. Assume $z_{n} \rightarrow z_{0}$. Finally, assume $Z_{0}$ does not hit $\mathcal{V}$. Then

$$
Z_{n} \Rightarrow Z_{0} \quad \text { weakly in } C\left([0, T], \mathbb{R}^{d}\right) \text { for every } T>0 .
$$

\section{Semimartingale reflected Brownian motion in a convex polyhedron}

\subsection{Definitions}

An open convex polyhedron $D$ is defined as follows. Fix $m \geq 1$, the number of edges. Let $\mathfrak{n}_{1}, \ldots, \mathfrak{n}_{m} \in \mathbb{R}^{d}$ be unit vectors, and let $b_{1}, \ldots, b_{m} \in \mathbb{R}$ be real numbers. The domain $D$ is defined as

$$
D=\left\{x \in \mathbb{R}^{d} \mid x \cdot \mathfrak{n}_{i}>b_{i}, i=1, \ldots, m\right\}
$$

We assume that $D \neq \varnothing$, and for each $j=1, \ldots, m$, we have:

$$
\left\{x \in \mathbb{R}^{d} \mid x \cdot \mathfrak{n}_{i}>b_{i}, i=1, \ldots, m, i \neq j\right\} \neq D .
$$

In this case, the edges of $D: D_{i}=\left\{x \in \bar{D} \mid \mathfrak{n}_{i} \cdot x=b_{i}\right\}, i=1, \ldots, m$, are $(d-1)$-dimensional. The vector $\mathfrak{n}_{i}$ is the inward unit normal vector to the face $D_{i}$, for each $i=1, \ldots, m$. The following subset of the boundary is called non-smooth parts of the boundary, and in our notation, it plays the role of the exceptional set $\mathcal{V}$ :

$$
\mathcal{V}=\bigcup_{1 \leq i<j \leq m}\left(D_{i} \cap D_{j}\right)
$$

We should note that $\mathcal{V}$ satisfies Assumption 1. The closure $\bar{D}$ of $D$ is called a closed convex polyhedron. In the sequel, we sometimes simply refer to $D$ or $\bar{D}$ as a convex polyhedron, if it is obvious from the context which one we are referring to.

Now, let us define an SRBM in the polyhedron $\bar{D}$, with drift vector $\mu \in \mathbb{R}^{d}$, covariance matrix $A$, and a $d \times m$ reflection matrix $R$. This is a continuous adapted process $Z=(Z(t), t \geq 0)$, which can be represented as

$$
Z(t)=W(t)+R L(t), \quad t \geq 0 .
$$


Here, $W=(W(t), t \geq 0)$ is a $d$-dimensional Brownian motion with drift vector $\mu$ and covariance matrix $A$, and $L=$ $\left(L_{1}, \ldots, L_{m}\right)^{\prime}$, where for each $i=1, \ldots, m, L_{i}=\left(L_{i}(t), t \geq 0\right)$ is a real-valued continuous nondecreasing adapted process with $L_{i}(0)=0$, which can increase only when $Z \in D_{i}$. This is denoted by $Z=\operatorname{SRBM}^{d}(\bar{D}, R, \mu, A)$. This is a process which reflects on each face $D_{i}, i=1, \ldots, m$, according to the vector $r_{i}$, the $i$ th column of the reflection matrix $R$. A particular case is an SRBM in the orthant $S=\mathbb{R}_{+}^{d}$, when $m=d, \mathfrak{n}_{i}=e_{i}$ is the standard $i$ th unit vector in $\mathbb{R}^{d}$, and $b_{i}=0$. Then $R$ is a $d \times d$-matrix, and the process $Z$ is denoted by $\operatorname{SRBM}^{d}(R, \mu, A)$.

An SRBM in a convex polyhedron, and, in particular, in the orthant, was a subject of extensive study over the past few decades. Existence and uniqueness results (weak and strong) are proved in $[4,6,16,20]$. For an SRBM in the orthant, see the survey [23].

An SRBM in a convex polyhedron fits into our general framework as follows: define the reflection field $r: \partial D \backslash$ $\mathcal{V} \rightarrow \mathbb{R}^{d}$ to be $r(x)=r_{i}$ for $x \in D_{i} \backslash \mathcal{V}, i=1, \ldots, m$. This function is continuous on $\partial D \backslash \mathcal{V}$. Sufficient conditions when an $\operatorname{SRBM}^{d}(\bar{D}, R, \mu, A)$ does not hit non-smooth parts of the boundary $\mathcal{V}$ are known: see [17,22]. Let us give an example.

Proposition 3.1. Consider a reflected Brownian motion $\operatorname{SRBM}^{d}(R, \mu, A)$ with $A=\left(a_{i j}\right)_{i, j=1, \ldots, d}$, and with $R=$ $\left(r_{i j}\right)_{i, j=1, \ldots, d}$ having $r_{i i}=1, i=1, \ldots, d ; r_{i j} \leq 0, i \neq j ;$ and the spectral radius of $I_{d}-R$ is strictly less than 1 . Then this SRBM a.s. does not hit non-smooth parts of the boundary if and only if

$$
r_{i j} a_{j j}+r_{j i} a_{i i} \geq 2 a_{i j}, \quad i, j=1, \ldots, d .
$$

\subsection{Main result}

The following result is a corollary of Theorem 2.7 .

Theorem 3.2. Take $m$ sequences of real numbers $\left(b_{i, n}\right)_{n \geq 0}, i=1, \ldots, m$. Take $m$ sequences of unit vectors in $\mathbb{R}^{d}$ : $\left(\mathfrak{n}_{i, n}\right)_{n \geq 0}, i=1, \ldots, m$. Assume that

$$
\mathfrak{n}_{i, n} \rightarrow \mathfrak{n}_{i, 0}, \quad b_{i, n} \rightarrow b_{i, 0}, \quad n \rightarrow \infty, \text { for each } i=1, \ldots, m .
$$

Consider a sequence $\left(D_{n}\right)_{n \geq 0}$ of convex polyhedra given by

$$
D_{n}=\left\{x \in \mathbb{R}^{d} \mid x \cdot \mathfrak{n}_{i, n}>b_{i, n}, i=1, \ldots, m\right\} .
$$

Take a sequence of positive definite symmetric $d \times d$ matrices $\left(A_{n}\right)_{n \geq 0}$ such that $A_{n} \rightarrow A_{0}$ as $n \rightarrow \infty$. Take a sequence $\left(g_{n}\right)_{n \geq 0}$ in $\mathbb{R}^{d}$ such that $g_{n} \rightarrow g_{0}$ as $n \rightarrow \infty$. Take a sequence of reflection matrices $\left(R_{n}\right)_{n \geq 0}$ such that $R_{n} \rightarrow R_{0}$. Assume that for every $n \geq 0$, the process $Z_{n}:=\operatorname{SRBM}^{d}\left(\bar{D}_{n}, R_{n}, g_{n}, A_{n}\right)$, starting from $Z_{n}(0)=z_{n} \in \bar{D}_{n}$, exists in the weak sense and is unique in law, and $z_{n} \rightarrow z_{0}$. Assume also that the process $Z_{0}$ does not hit non-smooth parts of the boundary $\partial D_{0}$. Then

$$
Z_{n} \Rightarrow Z_{0} \quad \text { weakly in } C\left([0, T], \mathbb{R}^{d}\right) \text { for every } T>0 .
$$

The proof is postponed until the next subsection. Let us give an application.

Example 4. Consider a fixed convex polyhedron $D \subseteq \mathbb{R}^{d}$. Let

$$
\mathcal{P}:=\left\{(R, A) \mid \operatorname{SRBM}^{d}(\bar{D}, R, \mu, A) \text { does not hit non-smooth parts of the boundary }\right\} .
$$

This definition makes sense because of the following fact: The property that an $\operatorname{SRBM}^{d}(\bar{D}, R, \mu, A)$ does not hit non-smooth parts of the boundary is independent of the starting point $z \in D$ and of the drift vector $\mu$. The proof of this independence statement is similar to that of [17, Proposition 3.3]. From Theorem 3.2, we can conclude that the process $\operatorname{SRBM}^{d}(\bar{D}, R, \mu, A)$, starting from $z \in D$, is continuous as an element of $C\left([0, T], \mathbb{R}^{d}\right)$, for every $T>0$, on the set

$$
\left\{(z, R, \mu, A) \mid z \in D,(R, A) \in \mathcal{P}, \mu \in \mathbb{R}^{d}\right\} .
$$




\subsection{Proof of Theorem 3.2}

We need to show that:

(i) $D_{n} \Rightarrow D_{0}$;

(ii) the condition (b) from Theorem 2.7 is satisfied;

(iii) $r_{n} \Rightarrow r_{0}$.

Proof of (i). We use Lemma 2.2(vii). Take a subsequence $\left(n_{k}\right)_{k \geq 1}$ and let $x_{n_{k}} \in \partial D_{n_{k}}$ be such that $x_{n_{k}} \rightarrow x_{0}$. Let us show that $x_{0} \in \partial D_{0}$. The boundary $\partial D_{n}$ for every $n$ consists of $m$ parts:

$$
\partial D_{n}=\bigcup_{i=1}^{m} D_{n, i}, \quad D_{n, i}:=\left\{x \in \mathbb{R}^{d} \mid \mathfrak{n}_{i, n} \cdot x=b_{i, n}, \mathfrak{n}_{j, n} \cdot x \geq b_{j, n}, j=1, \ldots, m, j \neq i\right\} .
$$

By the pigeonhole principle, there exists an $i_{0} \in\{1, \ldots, m\}$ and a subsequence $\left(n_{k}^{\prime}\right)_{k \geq 1} \subseteq\left(n_{k}\right)_{k \geq 1}$ such that $x_{n_{k}^{\prime}} \in$ $D_{n_{k}^{\prime}, i_{0}}$. That is,

$$
\mathfrak{n}_{i_{0}, n_{k}^{\prime}} \cdot x_{n_{k}^{\prime}}=b_{i_{0}, n_{k}^{\prime}}, \quad \mathfrak{n}_{j, n_{k}^{\prime}} \cdot x_{n_{k}^{\prime}} \geq b_{j, n_{k}^{\prime}}, \quad j=1, \ldots, m, j \neq i_{0} .
$$

Letting $k \rightarrow \infty$, we have: $\mathfrak{n}_{i_{0}, 0} \cdot x_{0}=b_{i_{0}, 0}$, and $\mathfrak{n}_{j, 0} \cdot x_{0} \geq b_{j, 0}, j=1, \ldots, m, j \neq i_{0}$. Therefore, $x_{0} \in D_{0, i_{0}} \subseteq \partial D_{0}$. Now, let us show (2). Take $x_{0} \in D_{0}$. Then $\mathfrak{n}_{i, 0} \cdot x_{0}>b_{i, 0}$, for $i=1, \ldots, m$. From (8), we get: there exists $n_{0}$ such that for $n>n_{0}$ we have: $\mathfrak{n}_{i, n} \cdot x_{0}>b_{i, n}, i=1, \ldots, m$. So $x_{0} \in D_{n}$ for $n>n_{0}$; therefore, $x_{0} \in \underline{\lim } D_{n}$. Similarly, if $x_{0} \in \bar{D}_{0}^{c}$, then there exists a $j \in\{1, \ldots, m\}$ such that $\mathfrak{n}_{j, 0} \cdot x_{0}<b_{j, 0}$. From (8), we get: there exists $n_{0}$ such that for $n>n_{0}$ we have: $\mathfrak{n}_{j, n} \cdot x_{0}<b_{j, n}$. Therefore, $x_{0} \in \bar{D}_{n}^{c}$ for $n>n_{0}$; so $x_{0} \in \underline{\lim } \bar{D}_{n}^{c}$. This completes the proof of (2).

Proof of (ii). We use Lemma 2.8. The domain $D_{n}$ has non-smooth parts of the boundary

$$
\mathcal{V}_{n}:=\bigcup_{1 \leq i<j \leq m} D_{n, i, j}
$$

where we denote

$$
D_{n, i, j}:=\left\{x \in \mathbb{R}^{d} \mid x \cdot \mathfrak{n}_{i, n}=b_{i, n}, x \cdot \mathfrak{n}_{j, n}=b_{j, n}, x \cdot \mathfrak{n}_{q, n} \geq b_{q, n}, q \neq i, j\right\} .
$$

Now, take a sequence $\left(x_{n_{k}}\right)_{k \geq 1}$ with $x_{n_{k}} \in \mathcal{V}_{n_{k}}$ and show that if $x_{n_{k}} \rightarrow x_{0}$, then $x_{0} \in \mathcal{V}_{0}$. By the pigeonhole principle, there exist a subsequence $\left(n_{k}^{\prime}\right)_{k \geq 1}$ and $1 \leq i<j \leq m$ such that $x_{n_{k}^{\prime}} \in D_{n_{k}^{\prime}, i, j}$. Therefore,

$$
x_{n_{k}^{\prime}} \cdot \mathfrak{n}_{i, n_{k}^{\prime}}=b_{i, n_{k}^{\prime}}, \quad x_{n_{k}^{\prime}} \cdot \mathfrak{n}_{j, n_{k}^{\prime}}=b_{j, n_{k}^{\prime}}, \quad x_{n_{k}^{\prime}} \cdot \mathfrak{n}_{q, n_{k}^{\prime}} \geq b_{q, n_{k}^{\prime}}, \quad q \neq i, j .
$$

Letting $k \rightarrow \infty$, we get:

$$
x_{0} \cdot \mathfrak{n}_{i, 0}=b_{i, 0}, \quad x_{0} \cdot \mathfrak{n}_{j, 0}=b_{j, n_{k}^{\prime}}, \quad x_{0} \cdot \mathfrak{n}_{q, n_{k}^{\prime}} \geq b_{q, n_{k}^{\prime}}, \quad q \neq i, j .
$$

Therefore, $x_{0} \in D_{0, i, j} \subseteq \mathcal{V}_{0}$. This completes the proof of (ii).

Proof of (iii). Take $x_{n} \in \partial D_{n} \backslash \mathcal{V}_{n}, n=0,1,2, \ldots$ such that $x_{n} \rightarrow x_{0}$. We need to prove that $r_{n}\left(x_{n}\right) \rightarrow r_{0}\left(x_{0}\right)$. Let us show that for every subsequence $\left(n_{k}\right)_{k \geq 1}$, there exists a subsequence $\left(n_{k}^{\prime}\right)_{k \geq 1} \subseteq\left(n_{k}\right)_{k \geq 1}$ such that

$$
r_{n_{k}^{\prime}}\left(x_{n_{k}^{\prime}}\right) \rightarrow r_{0}\left(x_{0}\right)
$$

Indeed, by the pigeonhole principle, there exists a $j \in\{1, \ldots, m\}$ and a subsequence $\left(n_{k}^{\prime}\right)_{k \geq 1}$ such that $x_{n_{k}^{\prime}} \in D_{n_{k}^{\prime}, j}$. Then, as discussed in the proof of (i) above, $x_{0} \in D_{0, j}$. Denote the $j$ th column of $R_{n}$ by $r_{n, j}$. Then $r_{n}(x) \equiv r_{n, j}$ for $x \in D_{n, j}$, by definition of a reflection field for an SRBM in a convex polyhedron. Now, $r_{n_{k}^{\prime}}\left(x_{n_{k}^{\prime}}\right)=r_{n_{k}^{\prime}, j} \rightarrow r_{0, j}=$ $r_{0}\left(x_{0}\right)$, because $R_{n} \rightarrow R_{0}$. This completes the proof. 


\section{Proof of Theorem 2.7}

\subsection{Outline of the proof}

For the rest of this section, fix a time horizon $T>0$. The first step is localization. Consider a compact set $\mathcal{K} \subseteq \mathbb{R}^{d} \backslash \mathcal{V}$ such that $z_{0} \in \operatorname{int} \mathcal{K}$. Let

$$
\tau_{\mathcal{K}, n}:=\inf \left\{t \geq 0 \mid Z_{n}(t) \notin \operatorname{int} \mathcal{K}\right\}, \quad n=0,1,2, \ldots
$$

Let $Z_{n}^{\mathcal{K}}(t) \equiv Z_{n}\left(t \wedge \tau_{\mathcal{K}, n}\right)$. We say that a continuous adapted process $\zeta=(\zeta(t), t \in[0, T])$ behaves as $\bar{Z}_{0}$ until it exits int $\mathcal{K}$ if for the stopping time

$$
\bar{\tau}_{\mathcal{K}, 0}:=\inf \{t \geq 0 \mid \zeta(t) \notin \mathcal{K}\},
$$

the process $\zeta\left(\cdot \wedge \bar{\tau}_{\mathcal{K}, 0}\right)$ has the same law as $Z_{0}^{\mathcal{K}}$. The following lemma was, in fact, already proved as Lemma 4.1 in [19].

Lemma 4.1. Assume that for every compact subset $\mathcal{K}$ as above every weak limit point of the sequence $\left(Z_{n}^{\mathcal{K}}\right)_{n \geq 1}$ in $C\left([0, T], \mathbb{R}^{d}\right)$ behaves as $\bar{Z}_{0}$ until it exits int $\mathcal{K}$. Then the conclusion of Theorem 2.7 is true.

Remark 3. If either (a) or (b) holds, then for every compact set $\mathcal{K} \subseteq \mathbb{R}^{d} \backslash \mathcal{V}_{0}$ there exists $n_{\mathcal{K}}$ such that for $n \geq n_{\mathcal{K}}$, we have: $Z_{n}^{\mathcal{K}}$ does not hit $\mathcal{V}_{n}$. Indeed, if (a) holds true, then there is nothing to prove. If (b) holds true, then $\operatorname{dist}\left(\mathcal{K}, \mathcal{V}_{0}\right):=$ $\varepsilon_{0}>0$, and there exists $n_{\mathcal{K}}$ such that for $n \geq n_{\mathcal{K}}$, we have:

$$
\max _{x \in \mathcal{V}_{n} \cap \mathcal{K}} \operatorname{dist}\left(x, \mathcal{V}_{0}\right)<\varepsilon_{0}
$$

In this case, for every $n \geq n_{\mathcal{K}}$ we have: $\mathcal{K} \cap \mathcal{V}_{n}=\varnothing$. Therefore, $Z_{n}^{\mathcal{K}}(t) \notin \mathcal{V}_{n}$ for these $n$ and for $t \in[0, T]$

The rest of the proof of Theorem 2.7 tracks the proofs from the paper [19].

Lemma 4.2. The sequence $\left(\varphi_{0}\left(Z_{n}^{\mathcal{K}}(\cdot)\right)\right)_{n \geq 1}$ is tight in $C[0, T]$.

Now, we can split $Z_{n}^{\mathcal{K}}$ into two components:

$$
Z_{n}^{\mathcal{K}}(t) \equiv Z_{n}\left(t \wedge \tau_{\mathcal{K}, n}\right)=V_{n}(t)+L_{n}(t),
$$

where for $n=1,2, \ldots$ and $t \in[0, T]$ we define:

$$
\begin{aligned}
& W_{n}^{\mathcal{K}}(t)=W_{n}\left(t \wedge \tau_{\mathcal{K}, n}\right), \\
& V_{n}(t):=z_{n}+\int_{0}^{t \wedge \tau_{\mathcal{K}, n}} g_{n}\left(Z_{n}(s)\right) \mathrm{d} s+\int_{0}^{t \wedge \tau_{\mathcal{K}, n}} \sigma_{n}\left(Z_{n}(s)\right) \mathrm{d} W_{n}(s), \\
& L_{n}(t):=\int_{0}^{t \wedge \tau_{\mathcal{K}, n}} r_{n}\left(Z_{n}(s)\right) \mathrm{d} l_{n}(s), \quad \text { and } \quad l_{n}^{\mathcal{K}}(t)=l_{n}\left(t \wedge \tau_{\mathcal{K}, n}\right),
\end{aligned}
$$

and $l_{n}$ is the process $l$ from Definition 1 for the reflected diffusion $Z_{n}$ in place of $Z$.

Lemma 4.3. The sequence $\left(V_{n}\right)_{n \geq 1}$ is tight in $C[0, T]$.

Lemma 4.4. The sequence $\left(l_{n}\right)_{n \geq 1}$ is tight in $C[0, T]$.

Lemma 4.5. The sequence $\left(L_{n}\right)_{n \geq 1}$ is tight in $C[0, T]$. 
The sequence $\left(W_{n}\right)_{n \geq 1}$ of Brownian motions is obviously tight in $C\left([0, T], \mathbb{R}^{d}\right.$ ) (all Brownian motions $W_{n}$ have the same distribution). Because each $W_{n}^{\mathcal{K}}$ is a Brownian motion $W_{n}$ stopped when it exits $\mathcal{K}$, the sequence $\left(W_{n}^{\mathcal{K}}\right)_{n \geq 1}$ is also tight in $C\left([0, T], \mathbb{R}^{d}\right)$. Using Lemmata 4.3, 4.4 and 4.5 , take a weak limit point $(\bar{V}, \bar{L}, \bar{l}, \bar{W})^{\prime}$ of the sequence

$$
\left(V_{n}, l_{n}^{\mathcal{K}}, L_{n}^{\mathcal{K}}, W_{n}^{\mathcal{K}}\right)^{\prime}
$$

We have: for some subsequence $\left(n_{k}\right)_{k \geq 1}$,

$$
\left(V_{n_{k}}, l_{n_{k}}^{\mathcal{K}}, L_{n_{k}}^{\mathcal{K}}, W_{n_{k}}^{\mathcal{K}}\right) \Rightarrow(\bar{V}, \bar{L}, \bar{l}, \bar{W})^{\prime} .
$$

By Skorohod representation theorem, see for example [9, Chapter 1], we can assume that the convergence is a.s. on a common probability space. From (9), we have:

$$
\bar{Z}(t):=\bar{V}(t)+\bar{L}(t)=\lim _{k \rightarrow \infty} Z_{n_{k}}^{\mathcal{K}}(t),
$$

where the convergence is uniform on $[0, T]$.

Lemma 4.6. The process $\bar{W}$ is a d-dimensional Brownian motion (with zero drift vector and identity covariance matrix), at least until the stopping time $\bar{\tau}_{\mathcal{K}}:=\inf \{t \geq 0 \mid \bar{Z}(t) \notin \operatorname{int} \mathcal{K}\}$. In addition, $\bar{\tau}_{\mathcal{K}} \leq \underline{\lim }_{k \rightarrow \infty} \tau_{\mathcal{K}, k}$ a.s.

Lemma 4.6 was proved as Lemma 4.5 in [19].

Lemma 4.7. For $t \in\left[0, \bar{\tau}_{\mathcal{K}}\right]$,

$$
\bar{V}(t)=z_{0}+\int_{0}^{t} g_{0}(\bar{Z}(s)) \mathrm{d} s+\int_{0}^{t} \sigma_{0}(\bar{Z}(s)) \mathrm{d} \bar{W}(s) .
$$

Now, let us state two lemmata which deal with the reflection terms.

Lemma 4.8. On the interval $\left[0, \bar{\tau}_{\mathcal{K}}\right]$, the process $\bar{l}$ is continuous, nondecreasing, can increase only when $\bar{Z} \in \partial D_{0}$, and $\bar{l}(0)=0$.

Lemma 4.9. For $t \in[0, T]$,

$$
\bar{L}(t)=\int_{0}^{t} r_{0}(\bar{Z}(s)) \mathrm{d} \bar{l}(s)
$$

Now, let us complete the proof of Theorem 2.7. Take a sequence $\left(m_{k}\right)_{k \geq 1}$. As in (10), there exists a subsequence $\left(n_{k}\right)_{k \geq 1}$ such that (10) holds. Combining the statements of Lemmata 4.6, 4.7, 4.8, 4.9, we get: for $t \leq \bar{\tau}_{\mathcal{K}}$,

$$
\bar{Z}(t)=\bar{V}(t)+\bar{L}(t)=z_{0}+\int_{0}^{t} g_{0}(\bar{Z}(s)) \mathrm{d} s+\int_{0}^{t} \sigma_{0}(\bar{Z}(s)) \mathrm{d} \bar{W}(s)+\int_{0}^{t} r_{0}(\bar{Z}(s)) \mathrm{d} \bar{l}(s),
$$

where $\bar{W}$ behaves as a Brownian motion until $\bar{\tau}_{\mathcal{K}}$, and the process $\bar{l}$ is continuous, nondecreasing, can increase only when $\bar{Z} \in \partial D$, and $\bar{l}(0)=0$. Therefore, $\bar{Z}$ behaves as $Z_{0}$ until it exits int $\mathcal{K}$. Apply Lemma 4.1 and finish the proof.

\subsection{Proof of Lemma 4.2}

The sequence of the processes $\left(Z_{n}^{\mathcal{K}}\right)_{n \geq 1}$ satisfy the following condition: for every $\delta>0$,

$$
\lim _{n \rightarrow \infty} \mathbf{P}\left(\min _{0 \leq t \leq T} \varphi_{0}\left(Z_{n}^{\mathcal{K}}(t)\right) \geq-\delta\right)=1 .
$$


This is analogous to [19, Lemma 4.2], but here it is much easier to prove. Indeed, $Z_{n}^{\mathcal{K}}(t) \in \bar{D}_{n} \cap \mathcal{K}$. But we know that $D_{n} \Rightarrow D_{0}$. From Lemma 2.2(iii), we get:

$$
\varliminf_{n \rightarrow \infty} \min _{x \in \bar{D}_{n} \cap \mathcal{K}} \varphi_{0}(x) \geq 0 .
$$

So there exists an $n_{0}(\delta)$ such that for $n \geq n_{0}(\delta)$ we have:

$$
\min _{x \in \bar{D}_{n} \cap \mathcal{K}} \varphi_{0}(x) \geq-\delta .
$$

Suppose that the following event happened:

$$
\left\{\omega\left(\varphi_{0}\left(Z_{n}^{\mathcal{K}}(\cdot)\right),[0, T], \varepsilon\right) \geq 3 \delta\right\} .
$$

Then there exist $t_{1}, t_{2} \in[0, T]$ such that $\varphi_{0}\left(Z_{n}^{\mathcal{K}}\left(t_{1}\right)\right)-\varphi_{0}\left(Z_{n}^{\mathcal{K}}\left(t_{2}\right)\right) \geq 3 \delta$ and $\left|t_{1}-t_{2}\right| \leq \varepsilon$. Let

$$
s_{1}:=t_{1} \wedge \tau_{\mathcal{K}, n}, \quad s_{2}:=t_{2} \wedge \tau_{\mathcal{K}, n} .
$$

Then $s_{1}, s_{2} \in\left[0, \tau_{\mathcal{K}, n}\right]$ and $\left|s_{1}-s_{2}\right| \leq \varepsilon$. Also, $\varphi_{0}\left(Z_{n}\left(s_{1}\right)\right)-\varphi_{0}\left(Z_{n}\left(s_{2}\right)\right) \geq 3 \delta$. Now, $\varphi_{0}\left(Z_{n}\left(s_{2}\right)\right) \geq-\delta$ because of (11). By continuity of $\varphi_{0}\left(Z_{n}(\cdot)\right)$, there exists $s_{0}$ between $s_{1}$ and $s_{2}$ such that

$$
\varphi_{0}\left(Z_{n}(s)\right) \geq \delta \quad \text { for } s \text { between } s_{1}, s_{0}, \quad \text { and } \quad \varphi_{0}\left(Z_{n}\left(s_{1}\right)\right)-\varphi_{0}\left(Z_{n}\left(s_{0}\right)\right) \geq \delta .
$$

Certainly, $\left|s_{0}-s_{1}\right| \leq \varepsilon$. But the function $\varphi_{0}$ is 1-Lipschitz, and so

$$
\left\|Z_{n}\left(s_{1}\right)-Z_{n}\left(s_{0}\right)\right\| \geq \varphi_{0}\left(Z_{n}\left(s_{1}\right)\right)-\varphi_{0}\left(Z_{n}\left(s_{0}\right)\right) \geq \delta .
$$

For $s \in\left[0, \tau_{\mathcal{K}, n}\right]$, we have: $Z_{n}(s) \in \mathcal{K}$. Since $\varphi_{0}\left(Z_{n}(s)\right) \geq \delta$ for $s$ between $s_{0}$ and $s_{1}$, we have: $Z_{n}\left(s_{1}\right)-Z_{n}\left(s_{0}\right)=$ $V_{n}\left(s_{1}\right)-V_{n}\left(s_{0}\right)$. Therefore,

$$
\left\|V_{n}\left(s_{1}\right)-V_{n}\left(s_{0}\right)\right\|=\left\|Z_{n}\left(s_{1}\right)-Z_{n}\left(s_{0}\right)\right\| \geq \delta .
$$

Taking $u_{1}=s_{1}, u_{2}=s_{0}$, we get from (13) that the following event actually happened:

$$
\left\{\omega\left(\varphi_{0}\left(Z_{n}^{\mathcal{K}}(\cdot)\right),[0, T], \varepsilon\right) \geq 3 \delta\right\} \subseteq A_{n}(\varepsilon),
$$

where we define

$$
A_{n}(\varepsilon):=\left\{\exists u_{1}, u_{2} \in[0, T]|| u_{1}-u_{2} \mid \leq \varepsilon,\left\|V_{n}\left(u_{1}\right)-V_{n}\left(u_{2}\right)\right\| \geq \delta\right\} .
$$

Now, the sequence $\left(V_{n}\left(\cdot \wedge \tau_{\mathcal{K}, n}\right)\right)_{n \geq 1}$ is tight. Indeed, we can write

$$
V_{n}\left(t \wedge \tau_{\mathcal{K}, n}\right)=z_{n}+\int_{0}^{t} g_{n}\left(Z_{n}(s)\right) 1_{\left\{s \leq \tau_{\mathcal{K}, n}\right\}} \mathrm{d} s+\int_{0}^{t} \sigma_{n}\left(Z_{n}(s)\right) 1_{\left\{s \leq \tau_{\mathcal{K}, n}\right\}} \mathrm{d} W_{n}(s) .
$$

Now, from Lemma 4.10 below, there exists $n_{1}$ such that for $n \geq n_{1}$,

$$
\left|g_{n}\left(Z_{n}(s)\right)\right| \leq C_{g}, \quad\left|\sigma_{n}\left(Z_{n}(s)\right)\right| \leq C_{\sigma}, \quad s \leq \tau_{\mathcal{K}, n} .
$$

Therefore, for all $s \in[0, T]$ and $n \geq n_{1}$,

$$
\left|g_{n}\left(Z_{n}(s)\right) 1_{\left\{s \leq \tau_{\mathcal{K}, n}\right\}}\right| \leq C_{g}, \quad\left|\sigma_{n}\left(Z_{n}(s)\right) 1_{\left\{s \leq \tau_{\mathcal{K}, n}\right\}}\right| \leq C_{\sigma} .
$$

By [18, Lemma 7.4] (applied to the local martingale part) and the Arzela-Ascoli criterion (applied to the bounded variation part), the sequence $\left(V_{n}\left(\cdot \wedge \tau_{\mathcal{K}, n}\right)\right)_{n \geq 1}$ is tight. Therefore,

$$
\lim _{\varepsilon \rightarrow 0} \sup _{n \geq 1} \mathbf{P}\left(\exists u_{1}, u_{2} \in[0, T]|| u_{1}-u_{2} \mid \leq \varepsilon,\left\|V_{n}\left(u_{1}\right)-V_{n}\left(u_{2}\right)\right\| \geq \delta\right)=0 .
$$


Comparing (15) with (14), we get:

$$
\limsup _{\varepsilon \rightarrow 0} \mathbf{P}\left(\omega\left(\varphi_{0}\left(Z_{n}^{\mathcal{K}}(\cdot)\right),[0, T], \varepsilon\right) \geq 3 \delta\right)=0 .
$$

Apply the Arzela-Ascoli criterion and complete the proof.

Lemma 4.10. There exists an $n_{0}$ and constants $C_{g}, C_{\sigma}, C_{r}$ such that for $n \geq n_{0}$, we have:

$$
\sup _{x \in \mathcal{K} \cap \bar{D}_{n}}\left\|g_{n}(x)\right\| \leq C_{g}, \quad \sup _{x \in \mathcal{K} \cap \bar{D}_{n}}\left\|\sigma_{n}(x)\right\| \leq C_{\sigma}, \quad \sup _{x \in \mathcal{K} \cap \partial D_{n}}\left\|r_{n}(x)\right\| \leq C_{r} .
$$

Proof. Let us prove this for $g_{n}$; the proofs for $\sigma_{n}$ and $r_{n}$ are similar. Assume the converse; then there exist $n_{k} \rightarrow \infty$ and $x_{n_{k}} \in \mathcal{K} \cap \bar{D}_{n_{k}}$ such that $\left\|g_{n_{k}}\left(x_{n_{k}}\right)\right\| \rightarrow \infty$. But the set $\mathcal{K}$ is compact, so there is a convergent subsequence $x_{n_{k}^{\prime}} \rightarrow x_{0} \in \mathcal{K} \cap \bar{D}_{0}$. Therefore, $g_{n_{k}^{\prime}}\left(x_{n_{k}^{\prime}}\right) \rightarrow g_{0}\left(x_{0}\right)$. This contradiction completes the proof.

\subsection{Proof of Lemma 4.3}

For all $s \geq 0, Z_{n}^{\mathcal{K}}(s) \in \mathcal{K} \cap \bar{D}_{n}$. We can conclude that the sequence

$$
t \mapsto \int_{0}^{t \wedge \tau_{\mathcal{K}, n}} g_{n}\left(Z_{n}^{\mathcal{K}}(s)\right) \mathrm{d} s
$$

is tight by Arzela-Ascoli criterion. Next, the sequence

$$
\bar{M}_{n}(t):=\int_{0}^{t \wedge \tau \mathcal{K}, n} \sigma_{n}\left(Z_{n}^{\mathcal{K}}(s)\right) \mathrm{d} W_{n}(s)
$$

is tight by [19, Lemma 6.4]. Indeed, each $\bar{M}_{n}$ is a continuous local martingale with $\bar{M}_{n}(0)=0$, and

$$
\left\langle\bar{M}_{n}\right\rangle_{t}=\int_{0}^{t \wedge \tau \mathcal{K}, n}\left\|\sigma_{n}\left(Z_{n}^{\mathcal{K}}(s)\right)\right\|^{2} \mathrm{~d} s .
$$

But $Z_{n}^{\mathcal{K}}(s) \in D_{n} \cap \mathcal{K}$ for all $s \in[0, T]$. Apply Lemma 4.10 and complete the proof.

\subsection{Proof of Lemma 4.4}

Let us state a technical lemma, which is proved in Appendix.

Lemma 4.11. For every compact subset $\mathcal{K} \subseteq \mathbb{R}^{d} \backslash \mathcal{V}_{0}$, there exists a $\delta_{\mathcal{K}} \in\left(0\right.$, $\left.\operatorname{dist}\left(\mathcal{K}, \mathcal{V}_{0}\right) / 2\right)$ such that:

(i) the signed distance function $\varphi_{0}$ is $C^{2}$ on the set

$$
\mathcal{K}^{\prime}:=\left\{x \in \mathcal{K}|| \varphi_{0}(x) \mid \equiv \operatorname{dist}\left(x, \partial D_{0}\right) \leq \delta_{\mathcal{K}}\right\} ;
$$

(ii) for every $x \in \mathcal{K}^{\prime}$, there exists a unique point $\zeta(x) \in \partial D_{0} \backslash \mathcal{V}_{0}$ which is the closest to $x$ on $\partial D_{0}$ : $\|x-\zeta(x)\|=$ $\operatorname{dist}\left(x, \partial D_{0}\right)=\operatorname{dist}\left(x, \partial D_{0} \backslash \mathcal{V}_{0}\right)$, and this function $\zeta$ is continuous on $\mathcal{K}^{\prime}$.

Take a $C^{\infty}$ function $\psi: \mathbb{R} \rightarrow \mathbb{R}$ such that

$$
\psi(x):= \begin{cases}x, & |x| \leq \delta_{\mathcal{K}} / 2 \\ 0, & |x| \geq \delta_{\mathcal{K}} .\end{cases}
$$

Let us write an Itô equation for the process $\psi\left(\varphi_{0}\left(Z_{n}^{\mathcal{K}}(\cdot)\right)\right)$, or, equivalently, for $\left(\psi \circ \varphi_{0}\right)\left(Z_{n}(t)\right)$ for $t \leq \tau_{\mathcal{K}, n}$. We have: $\psi \circ \varphi_{0} \in C^{2}$ on $\mathcal{K}$. Therefore, we can apply Itô formula for the function $\psi \circ \varphi_{0}$. We have: $\nabla\left(\psi \circ \varphi_{0}\right)(x)=$ 
$\psi^{\prime}\left(\varphi_{0}(x)\right) \nabla \varphi_{0}(x)$. Abusing the notation, we can write this even if $\left|\varphi_{0}(x)\right|>\delta_{\mathcal{K}}$, where the function $\varphi_{0}$ might not be $C^{2}$, since then $\psi^{\prime}\left(\varphi_{0}(x)\right)=0$ and the left-hand side is also zero. In addition, a similar formula holds for second derivatives:

$$
\theta_{i j}(x):=\frac{\partial^{2}\left(\psi \circ \varphi_{0}\right)(x)}{\partial x_{i} \partial x_{j}}=\psi^{\prime \prime}\left(\varphi_{0}(x)\right) \frac{\partial \varphi_{0}}{\partial x_{i}} \frac{\partial \varphi_{0}}{\partial x_{j}}+\psi^{\prime}\left(\varphi_{0}(x)\right) \frac{\partial^{2} \varphi_{0}}{\partial x_{i} \partial x_{j}} .
$$

By Itô's formula, for $t \leq \tau_{\mathcal{K}, n}$,

$$
\mathrm{d} \psi\left(\varphi_{0}\left(Z_{n}(t)\right)\right)=\psi^{\prime}\left(\varphi_{0}\left(Z_{n}(t)\right)\right) \nabla \varphi_{0}\left(Z_{n}(t)\right) \cdot \mathrm{d} Z_{n}(t)+\sum_{i=1}^{d} \sum_{j=1}^{d} \theta_{i j}\left(Z_{n}(t)\right) \mathrm{d}\left\langle\left(Z_{n}\right)_{i},\left(Z_{n}\right)_{j}\right\rangle_{t} .
$$

Now, from (9) and the fact that $L_{n}$ has finite variation, we get: for $t \leq \tau_{\mathcal{K}, n}$,

$$
\mathrm{d}\left\langle\left(Z_{n}\right)_{i},\left(Z_{n}\right)_{j}\right\rangle_{t}=\left(\sigma_{n} \sigma_{n}^{T}\right)_{i j}\left(Z_{n}(t)\right) \mathrm{d} t
$$

From the properties of $\varphi_{0}$ and $\psi$ it follows that the function $\psi^{\prime}\left(\varphi_{0}(x)\right) \nabla \varphi_{0}(x)$, as well as each $\theta_{i j}$ is bounded on $\mathcal{K}$. Apply Lemma 4.10 and note that $Z_{n}(t) \in \bar{D}_{n} \cap \mathcal{K}$ for $t \leq \tau_{\mathcal{K}, n}$. By the Arzela-Ascoli criterion, the following sequence is tight:

$$
t \mapsto \int_{0}^{t \wedge \tau_{\mathcal{K}, n}} \sum_{i=1}^{d} \sum_{j=1}^{d} \theta_{i j}\left(Z_{n}(t)\right) \mathrm{d}\left\langle\left(Z_{n}\right)_{i},\left(Z_{n}\right)_{j}\right\rangle_{t}
$$

Take the first term in the right-hand side of (18)

$$
\begin{aligned}
\psi^{\prime}\left(\varphi_{0}\left(Z_{n}(t)\right)\right) \nabla \varphi_{0}\left(Z_{n}(t)\right) \cdot \mathrm{d} Z_{n}(t)= & \psi^{\prime}\left(\varphi_{0}\left(Z_{n}(t)\right)\right) \nabla \varphi_{0}\left(Z_{n}(t)\right) \cdot g_{n}\left(Z_{n}(t)\right) \mathrm{d} t \\
& +\psi^{\prime}\left(\varphi_{0}\left(Z_{n}(t)\right)\right) \nabla \varphi_{0}\left(Z_{n}(t)\right) \cdot \sigma_{n}\left(Z_{n}(t)\right) \mathrm{d} W_{n}(t) \\
& +\psi^{\prime}\left(\varphi_{0}\left(Z_{n}(t)\right)\right) \nabla \varphi_{0}\left(Z_{n}(t)\right) \cdot r_{n}\left(Z_{n}(t)\right) \mathrm{d} l_{n}(t)
\end{aligned}
$$

By Lemma 4.10 and the Arzela-Ascoli criterion, the following sequence is tight:

$$
t \mapsto \int_{0}^{t \wedge \tau_{\mathcal{K}, n}} \psi^{\prime}\left(\varphi_{0}\left(Z_{n}(s)\right)\right) \nabla \varphi_{0}\left(Z_{n}(s)\right) \cdot g_{n}\left(Z_{n}(s)\right) \mathrm{d} s
$$

Next, the following sequence of continuous local martingales

$$
M_{n}(t):=\int_{0}^{t \wedge \tau_{\mathcal{K}, n}} \psi^{\prime}\left(\varphi_{0}\left(Z_{n}(s)\right)\right) \nabla \varphi_{0}\left(Z_{n}(s)\right) \cdot \sigma_{n}\left(Z_{n}(s)\right) \mathrm{d} W_{n}(s)
$$

is tight by Lemma 6.4 from [19]. Indeed,

$$
\left\langle M_{n}\right\rangle_{t}=\int_{0}^{t \wedge \tau_{\mathcal{K}, n}} \psi^{\prime 2}\left(\varphi_{0}\left(Z_{n}(s)\right)\right)\left\|\nabla \varphi_{0}\left(Z_{n}(s)\right) \cdot \sigma_{n}\left(Z_{n}(s)\right)\right\|^{2} \mathrm{~d} s,
$$

and the derivative of this function with respect to $t$ is uniformly bounded. (This follows from the fact that $\psi^{\prime}$ is bounded on $\mathbb{R}, \varphi_{0}$ is bounded on $\mathcal{K}$, and from Lemma 4.10. By Lemma 6.7 from the same article [19], the sequence $\psi\left(\varphi_{0}\left(Z_{n}^{\mathcal{K}}(\cdot)\right)\right)$ is itself tight. Therefore, the sequence

$$
N_{n}(t):=\int_{0}^{t \wedge \tau_{\mathcal{K}, n}} \psi^{\prime}\left(\varphi_{0}\left(Z_{n}(s)\right)\right) \nabla \varphi_{0}\left(Z_{n}(s)\right) \cdot r_{n}\left(Z_{n}(s)\right) \mathrm{d} l_{n}(s)
$$


is tight in $C\left([0, T], \mathbb{R}^{d}\right)$. But the process $l_{n}$ can grow only when $Z_{n} \in \partial D_{0}$, that is, when $\varphi_{0}\left(Z_{n}(s)\right)=0$. For these $s$ we have: $\psi^{\prime}\left(\varphi_{0}\left(Z_{n}(s)\right)\right)=1$, because $\psi^{\prime}(0)=1$. Therefore, we can rewrite

$$
N_{n}(t):=\int_{0}^{t \wedge \tau_{\mathcal{K}, n}} \nabla \varphi_{0}\left(Z_{n}(s)\right) \cdot r_{n}\left(Z_{n}(s)\right) \mathrm{d} l_{n}(s) .
$$

Lemma 4.12. There exists $n_{0}$ and $\varepsilon_{0}>0$ such that for $n \geq n_{0}$, for $x \in \partial \bar{D}_{n} \cap \mathcal{K}$, we have: $\nabla \varphi_{0}(x) \cdot r_{n}(x) \geq \varepsilon_{0}$.

Proof. Assume the converse. Then there exist a subsequence $\left(n_{k}\right)_{k \geq 1}$ and a corresponding sequence of points $x_{n_{k}} \in$ $\partial \bar{D}_{n_{k}} \cap \mathcal{K}$ such that

$$
\nabla \varphi_{0}\left(x_{n_{k}}\right) \cdot r_{n_{k}}\left(x_{n_{k}}\right) \leq \frac{1}{k}
$$

Since $\mathcal{K}$ is compact, there exists a subsequence $\left(n_{k}^{\prime}\right)_{k \geq 1}$ such that $x_{n_{k}^{\prime}} \rightarrow x_{0}$. Then $x_{0} \in \mathcal{K} \cap \partial D_{0}$. For all $k \geq k_{0}$, $x_{n_{k}^{\prime}} \in \mathcal{K}^{\prime}$ (and $x_{0} \in \mathcal{K}^{\prime}$ ). But $\nabla \varphi_{0}$ is continuous on $\mathcal{K}^{\prime}$. Therefore, $\nabla \varphi_{0}\left(x_{n_{k}}\right) \rightarrow \nabla \varphi_{0}\left(x_{0}\right)$. Also, since $r_{n} \Rightarrow r_{0}$, we have: $r_{n_{k}}\left(x_{n_{k}}\right) \rightarrow r_{0}\left(x_{0}\right)$. Therefore, passing to the limit, we have: $\nabla \varphi_{0}\left(x_{0}\right) \cdot r_{0}\left(x_{0}\right) \leq 0$. But $\nabla \varphi_{0}\left(x_{0}\right)$ has the same direction as the inward unit normal vector $\mathfrak{n}\left(x_{0}\right)$ to $\partial D_{0}$, and by the properties of the reflection field $r_{0}$ we have: $\mathfrak{n}\left(x_{0}\right) \cdot r_{0}\left(x_{0}\right)>0$. This contradiction completes the proof.

In view of Lemma 4.12, we can rewrite (19) as

$$
l_{n}\left(t \wedge \tau_{\mathcal{K}, n}\right):=\int_{0}^{t \wedge \tau_{\mathcal{K}, n}}\left[\nabla \varphi_{0}\left(Z_{n}(s)\right) \cdot r_{n}\left(Z_{n}(s)\right)\right]^{-1} \mathrm{~d} N_{n}(t) .
$$

But $\left(N_{n}\right)_{n \geq 1}$ is tight, and by Lemma 4.12 we have:

$$
\left[\nabla \varphi_{0}\left(Z_{n}(s)\right) \cdot r_{n}\left(Z_{n}(s)\right)\right]^{-1} \leq \varepsilon_{0}^{-1} .
$$

Therefore, $l_{n}\left(\cdot \wedge \tau_{\mathcal{K}, n}\right)$ is tight. The proof is complete.

\subsection{Proof of Lemma 4.5}

Note that the process $l_{n}$ can grow only when $Z_{n} \in \partial D_{n}$. By Lemma 4.10, for $n \geq n_{0}$,

$$
\sup _{0 \leq s \leq t \wedge \tau_{\mathcal{K}, n}}\left\|r_{n}\left(Z_{n}(s)\right)\right\| \leq C_{r}
$$

Therefore, the sequence $\left(L_{n}\right)_{n \geq 1}$ is also tight.

\subsection{Proof of Lemma 4.7}

Without loss of generality, assume $n_{k}=k$ for convenience of notation. We have: $Z_{k}^{\mathcal{K}} \rightarrow \bar{Z}$ uniformly on [0,T], and

$$
Z_{k}^{\mathcal{K}}(s) \in \bar{D}_{k} \cap \mathcal{K}, \quad \text { and } \quad \bar{Z}(s) \in \bar{D}_{0} \cap \mathcal{K} \quad \text { for } s \in[0, T] .
$$

Recall the definition of locally uniform convergence of functions defined on different subsets of $\mathbb{R}^{d}$. Since $g_{n} \Rightarrow g_{0}$, $\sigma_{n} \Rightarrow \sigma_{0}$ by Remark 2 , and

$$
Z_{k}^{\mathcal{K}}(s) \rightarrow \bar{Z}(s) \quad \text { uniformly on }[0, T],
$$

by Lemma 2.2(v) we have:

$$
g_{k}\left(Z_{k}^{\mathcal{K}}(s)\right) \rightarrow g_{0}(\bar{Z}(s)), \quad \sigma_{k}\left(Z_{k}^{\mathcal{K}}(s)\right) \rightarrow \sigma_{0}(\bar{Z}(s)) .
$$


From Lemma 4.14, we have:

$$
\int_{0}^{t \wedge \tau \mathcal{K}, k} \sigma_{k}\left(Z_{k}^{\mathcal{K}}(s)\right) \mathrm{d} W_{k}(s)=\int_{0}^{t} \sigma_{k}\left(Z_{k}^{\mathcal{K}}(s)\right) \mathrm{d} W_{k}^{\mathcal{K}} \rightarrow \int_{0}^{t} \sigma_{0}(\bar{Z}(s)) \mathrm{d} \bar{W}(s),
$$

where the convergence is understood in probability. Therefore, there exists a subsequence $\left(k_{m}\right)_{m \geq 1}$ such that

$$
\int_{0}^{t \wedge \tau_{\mathcal{K}, k_{m}}} \sigma_{k_{m}}\left(Z_{k_{m}}^{\mathcal{K}}(s)\right) \mathrm{d} W_{k_{m}}(s) \rightarrow \int_{0}^{t} \sigma_{0}(\bar{Z}(s)) \mathrm{d} \bar{W}(s) \quad \text { a.s. uniformly on }[0, T] .
$$

Lemma 4.13. Uniformly on $\left[0, \bar{\tau}_{\mathcal{K}}\right]$, we have:

$$
\int_{0}^{t \wedge \tau \mathcal{K}, n} g_{k}\left(Z_{k}^{\mathcal{K}}(s)\right) \mathrm{d} s \rightarrow \int_{0}^{t \wedge \bar{\tau} \mathcal{K}} g_{0}(\bar{Z}(s)) \mathrm{d} s .
$$

Proof. For every $\varepsilon>0$ there exists $k_{1}(\varepsilon)$ such that for $k \geq k_{1}(\varepsilon)$ we have: $\tau_{\mathcal{K}, k} \leq \bar{\tau}_{\mathcal{K}}+\varepsilon$, and so for $t \leq \bar{\tau}_{\mathcal{K}}$ we have: $\left|t \wedge \bar{\tau}_{\mathcal{K}}-t \wedge \tau_{\mathcal{K}, k}\right| \leq \varepsilon$. Therefore,

$$
\left|\int_{0}^{t \wedge \bar{\tau} \mathcal{K}} g_{0}(\bar{Z}(s)) \mathrm{d} s-\int_{0}^{t \wedge \tau_{\mathcal{K}, k}} g_{0}(\bar{Z}(s)) \mathrm{d} s\right| \leq \varepsilon \cdot \max _{[0, T]}\left|g_{0}(\bar{Z}(s))\right| .
$$

From (20), we have: $g_{k}\left(Z_{k}^{\mathcal{K}}(t)\right) \rightarrow g_{0}(\bar{Z}(t))$ uniformly on $[0, T]$. Therefore, there exists $n_{\varepsilon}$ such that for $n \geq n_{\varepsilon}$ we have:

$$
\max _{t \in[0, T]}\left\|g_{n}\left(Z_{n}^{\mathcal{K}}(t)\right)-g_{0}(\bar{Z}(t))\right\| \leq \varepsilon
$$

We have: for $n \geq n_{\varepsilon}$,

$$
\left|\int_{0}^{t \wedge \tau_{\mathcal{K}, n}} g_{n}\left(Z_{n}^{\mathcal{K}}(s)\right) \mathrm{d} s-\int_{0}^{t \wedge \tau \mathcal{K}, n} g_{0}(\bar{Z}(s)) \mathrm{d} s\right| \leq T \cdot \max _{t \in[0, T]}\left\|g_{n}\left(Z^{\mathcal{K}}(t)\right)-g_{0}(\bar{Z}(t))\right\| \leq T \varepsilon .
$$

Combining (23) and (24), we have: for $n \geq n_{\varepsilon}$,

$$
\left|\int_{0}^{t \wedge \bar{\tau} \mathcal{K}} g_{0}(\bar{Z}(s)) \mathrm{d} s-\int_{0}^{t \wedge \tau_{\mathcal{K}, n}} g_{n}\left(Z_{n}^{\mathcal{K}}(s)\right) \mathrm{d} s\right| \leq \varepsilon \cdot\left(T+\max _{[0, T]}\left|g_{0}(\bar{Z}(s))\right|\right) .
$$

Since $\varepsilon>0$ is arbitrary, the proof is complete.

Combining Lemma 4.13 with (22) and $z_{n} \rightarrow z_{0}$, we get: uniformly on $\left[0, \bar{\tau}_{\mathcal{K}}\right]$,

$$
\begin{aligned}
V_{k_{m}}(t) & =z_{k_{m}}+\int_{0}^{t \wedge \tau_{\mathcal{K}, k_{m}}} g_{k_{m}}\left(Z_{k_{m}}^{\mathcal{K}}(s)\right) \mathrm{d} s+\int_{0}^{t \wedge \tau_{\mathcal{K}, k_{m}}} \sigma_{k_{m}}\left(Z_{k_{m}}^{\mathcal{K}}(s)\right) \mathrm{d} W_{k_{m}}(s) \\
& \rightarrow z_{0}+\int_{0}^{t \wedge \bar{\tau} \mathcal{K}} g_{0}(\bar{Z}(s)) \mathrm{d} s+\int_{0}^{t \wedge \bar{\tau}_{\mathcal{K}}} \sigma_{0}(\bar{Z}(s)) \mathrm{d} W_{0}(s) .
\end{aligned}
$$

But $V_{n} \rightarrow V_{0}$ a.s. uniformly on $[0, T]$. This completes the proof of Lemma 4.7.

Lemma 4.14. For $m \geq 1$, let $Y_{k}=\left(Y_{k}(t), 0 \leq t \leq T\right)$ be an $\mathbb{R}^{d}$-valued continuous adapted process, and let $U_{k}=$ $\left(U_{k}(t), 0 \leq t \leq T\right)$ be an $\mathbb{R}^{d}$-valued continuous local martingale. If in $C\left([0, T], \mathbb{R}^{d} \times \mathbb{R}^{d}\right)$ we have: $\left(Y_{k}, U_{k}\right) \Rightarrow$ $(Y, U), k \rightarrow \infty$, then $U$ is a semimartingale, and we have the following convergence in probability:

$$
\int_{0}^{t} Y_{k} \mathrm{~d} U_{k} \rightarrow \int_{0}^{t} Y \mathrm{~d} U \quad \text { uniformly on } t \in[0, T] .
$$

This lemma was proved in [12, Theorem 5.10]; see also [8, Lemma 3.6]. Both of these statements are more general than Lemma 4.14. For convenience, we state this result here in the form which is convenient for our use. 


\subsection{Proof of Lemma 4.8}

As before, assume for simplicity that $n_{k}=k$. Fix $\varepsilon>0$ and let us prove these properties for $\bar{l}$ on $\left[0, \bar{\tau}_{\mathcal{K}}-\varepsilon\right]$. Note that there exists $n(\varepsilon)$ such that for $k \geq n(\varepsilon)$ we have: $\bar{\tau}_{\mathcal{K}} \leq \tau_{\mathcal{K}, k}+\varepsilon$. Now, $l_{k}^{\mathcal{K}} \rightarrow \bar{l}(t)$ uniformly on [0,T]; but $l_{k}^{\mathcal{K}}(t) \equiv l_{k}\left(t \wedge \tau_{\mathcal{K}, k}\right) \equiv l_{k}(t)$ for $t \in\left[0, \tau_{\mathcal{K}, k}\right] \subseteq\left[0, \bar{\tau}_{\mathcal{K}}-\varepsilon\right]$. Now, $l_{k}$ is nondecreasing and $l_{k}(0)=0$; therefore, the same properties hold for $\bar{l}$ on $\left[0, \bar{\tau}_{\mathcal{K}}-\varepsilon\right]$.

Fix $\delta>0$ and let us show that $\bar{l}$ does not increase on $\left[t_{1}, t_{2}\right] \subseteq\left[0, \bar{\tau}_{\mathcal{K}}-\varepsilon\right]$ if $\operatorname{dist}\left(\bar{Z}(t), \partial D_{0}\right)>\delta$ for $t \in\left[t_{1}, t_{2}\right]$. Indeed, since $Z_{k}^{\mathcal{K}} \rightarrow \bar{Z}$ uniformly on $[0, T]$, by Lemma $2.2(\mathrm{v})$ we have:

$$
\varphi_{k}\left(Z_{k}\right) \rightarrow \varphi_{0}(\bar{Z}) \quad \text { uniformly on }\left[t_{1}, t_{2}\right] .
$$

$\operatorname{But} \operatorname{dist}\left(Z_{k}(t), \partial D_{k}\right) \equiv\left|\varphi_{k}\left(Z_{k}(t)\right)\right|$. Therefore,

$$
\operatorname{dist}\left(Z_{k}(t), \partial D_{k}\right) \rightarrow \operatorname{dist}\left(\bar{Z}(t), \partial D_{0}\right) \quad \text { uniformly on }\left[t_{1}, t_{2}\right] \text {. }
$$

Therefore, for $k \geq m(\delta), t \in\left[t_{1}, t_{2}\right]$, we have: $\operatorname{dist}\left(Z_{k}(t), \partial D_{k}\right) \geq \delta / 2$. Meanwhile, $l_{k}$ does not grow on $\left[t_{1}, t_{2}\right]$ : that is, $l_{k}\left(t_{1}\right)=l_{k}\left(t_{2}\right)$. Let $k \rightarrow \infty$ and conclude: $\bar{l}\left(t_{1}\right)=\bar{l}\left(t_{2}\right)$. Thus, $\bar{l}$ does not grow on $\left[t_{1}, t_{2}\right]$.

Now, let us prove a more general statement: if $\left[t_{1}, t_{2}\right] \subseteq\left[0, \bar{\tau}_{\mathcal{K}}\right]$ and dist $\left(\bar{Z}(t), \partial D_{0}\right)>0$ for $t \in\left[t_{1}, t_{2}\right]$, then $\bar{l}\left(t_{1}\right)=$ $\bar{l}\left(t_{2}\right)$. Indeed, assume $\bar{l}\left(t_{1}\right)<\bar{l}\left(t_{2}\right)$. By continuity of $\bar{l}$, there exists $\varepsilon>0$ such that $\bar{l}\left(t_{1}\right)<\bar{l}\left(t_{2}-\varepsilon\right)$. By continuity of $\bar{Z}$, there exists $\delta>0$ such that $\operatorname{dist}\left(\bar{Z}(t), \partial D_{0}\right) \geq \delta$ for $t \in\left[t_{1}, t_{2}\right]$. Now, repeat the previous argument and conclude: $\bar{l}\left(t_{1}\right)=\bar{l}\left(t_{2}-\varepsilon\right)$. This contradiction completes the proof.

\subsection{Proof of Lemma 4.9}

As before, we assume $n_{k}=k$ without loss of generality. There exists $n_{0}$ such that for $n \geq n_{0}$, we have: for $x \in \partial D_{n} \cap \mathcal{K}$, $\left|\varphi_{0}(x)\right| \leq \delta_{\mathcal{K}}$. This follows from Lemma 2.2. In other words, for $n \geq n_{0}$ we have: $\partial D_{n} \cap \mathcal{K} \subseteq \mathcal{K}^{\prime}$, where $\mathcal{K}^{\prime}$ was defined in (17). By Lemma 4.11(ii), the distance function $\zeta$ is continuous on $\mathcal{K}^{\prime}$. Note that

$$
\varepsilon_{n}:=\max _{x \in \mathcal{K} \cap \partial D_{n}}\left|\varphi_{0}(x)\right| \rightarrow 0 .
$$

For $x \in \mathcal{K}_{0}$, we have: $\|\zeta(x)-x\|=\operatorname{dist}\left(x, \partial D_{0}\right)=\left|\varphi_{0}(x)\right| \leq \varepsilon_{n}$. Therefore, by definition of locally uniform convergence $r_{n} \Rightarrow r_{0}$, we have:

$$
\sup _{x \in \mathcal{K} \cap \partial D_{n}}\left\|r_{n}(x)-r_{0}(\zeta(x))\right\| \rightarrow 0
$$

Therefore, we get:

$$
\begin{aligned}
& \int_{0}^{t} r_{k}\left(Z_{k}^{\mathcal{K}}(s)\right) \mathrm{d} l_{k}^{\mathcal{K}}(s)-\int_{0}^{t} r_{0}(\bar{Z}(s)) \mathrm{d} \bar{l}(s)=I_{1}(k)+I_{2}(k)+I_{3}(k), \\
& I_{1}(k):=\int_{0}^{t}\left[r_{k}\left(Z_{k}^{\mathcal{K}}(s)\right)-r_{0}\left(\zeta\left(Z_{k}^{\mathcal{K}}(s)\right)\right)\right] \mathrm{d} l_{k}^{\mathcal{K}}(s), \\
& I_{2}(k):=\int_{0}^{t}\left[r_{0}\left(\zeta\left(Z_{k}^{\mathcal{K}}(s)\right)\right)-r_{0}(\bar{Z}(s))\right] \mathrm{d} \bar{l}(s), \\
& I_{3}(k):=\int_{0}^{t} r_{0}\left(\zeta\left(Z_{k}^{\mathcal{K}}(s)\right)\right) \mathrm{d} l_{k}^{\mathcal{K}}(s)-\int_{0}^{t} r_{0}\left(\zeta\left(Z_{k}^{\mathcal{K}}(s)\right)\right) \mathrm{d} \bar{l}(s) .
\end{aligned}
$$

By Lemma 6.2 from [19], $\left\|I_{1}(k)\right\| \rightarrow 0$ as $k \rightarrow \infty$. From the relation (25), the fact that each $l_{k}^{\mathcal{K}}$ is nondecreasing, and the convergence $l_{k}^{\mathcal{K}}(T) \rightarrow \bar{l}(T)$, we have: $\left\|I_{3}(k)\right\| \rightarrow 0$. Finally, $\zeta(\bar{Z}(s))=\bar{Z}(s)$ when $\bar{Z}(s) \in \partial D_{0}$. But the function $\bar{l}$ can grow only when $\bar{Z}(s) \in \partial D_{0}$ : this follows from Lemma 4.8. Therefore,

$$
\int_{0}^{t} r_{0}(\bar{Z}(s)) \mathrm{d} \bar{l}(s)=\int_{0}^{t} r_{0}(\zeta(\bar{Z}(s))) \mathrm{d} \bar{l}(s)
$$


The function $\zeta$ is continuous on $\mathcal{K}_{0}$, and since $Z_{k}^{\mathcal{K}} \rightarrow \bar{Z}$ uniformly on $[0, T]$, we have: $\zeta\left(Z_{k}^{\mathcal{K}}\right) \rightarrow \zeta(\bar{Z})$ uniformly on $[0, T]$. Therefore, as $k \rightarrow \infty$,

$$
\left\|I_{2}(k)\right\| \leq \max _{0 \leq s \leq T}\left\|r_{0}(\bar{Z}(s))-r_{0}\left(\zeta\left(Z_{k}^{\mathcal{K}}(s)\right)\right)\right\| \cdot \bar{l}(T) \rightarrow 0 .
$$

\section{Convergence to a non-reflected diffusion}

\subsection{Convergence of domains to the whole space}

Let us modify the definition of weak convergence $D_{n} \Rightarrow D_{0}$ for the case $D_{0}=\mathbb{R}^{d}$. The main question is how to define $\varphi_{0}(x)$, the signed distance from $x \in \mathbb{R}^{d}$ to the boundary $\partial D_{0}$, because the set $D_{0}=\mathbb{R}^{d}$ has no boundary: $\partial \mathbb{R}^{d}=\varnothing$. Intuitively, we can approximate $\mathbb{R}^{d}$ by a very large ball $U(0, r)$. Take a point $x \in \mathbb{R}^{d}$. Since $r$ is large, $x \in U(0, r)$, and the distance from $x$ to $\partial U(0, r)$ is equal to $r-\|x\|$, which is also large. Therefore, it makes sense to define $\varphi_{0}(x):=\infty$ for all $x \in \mathbb{R}^{d}$.

Definition 5. We say that a sequence of domains $\left(D_{n}\right)_{n \geq 1}$ converges weakly to $\mathbb{R}^{d}$ and write $D_{n} \Rightarrow \mathbb{R}^{d}$, if $\varphi_{n}(x) \rightarrow \infty$ for all $x \in \mathbb{R}^{d}$.

The following is an equivalent characterization of this weak convergence. The proof is postponed until Appendix.

Lemma 5.1. $D_{n} \Rightarrow \mathbb{R}^{d}$ if and only if for every compact set $\mathcal{K} \subseteq \mathbb{R}^{d}$ there exists an $n_{0}$ such that for $n>n_{0}$ we have: $\mathcal{K} \subseteq D_{n}$

Let us state an analogue of Theorem 2.7 for the case when $D_{n} \Rightarrow \mathbb{R}^{d}$, In this case, reflected diffusions $Z_{n}$ converge weakly to a non-reflected diffusion $Z_{0}$ in $\mathbb{R}^{d}$. Take a sequence $\left(D_{n}\right)_{n \geq 1}$ of domains in $\mathbb{R}^{d}$. For each $n \geq 1$, consider a reflected diffusion $Z_{n}=\left(Z_{n}(t), t \geq 0\right)$ in $\bar{D}_{n}$ with drift vector $g_{n}(\cdot)$, covariance matrix $A_{n}(\cdot)$, and reflection field $r_{n}(\cdot)$, starting from $Z_{n}(0)=z_{n}$. We suppose that Assumptions 1, 2, 3 are satisfied. We do not impose a condition that $Z_{n}$ does not hit non-smooth parts $\mathcal{V}_{n}$ of the boundary $\partial D_{n}$. Define a drift coefficient $g_{0}: \mathbb{R}^{d} \rightarrow \mathbb{R}^{d}$ and a covariance matrix $A_{0}: \mathbb{R}^{d} \rightarrow \mathcal{P}_{d}$. For each $x \in \mathbb{R}^{d}$, let $\sigma_{0}(x)=A^{1 / 2}(x)$. Consider a non-reflected diffusion process

$$
\mathrm{d} Z_{0}(t)=g_{0}\left(Z_{0}(t)\right) \mathrm{d} t+\sigma_{0}\left(Z_{0}(t)\right) \mathrm{d} W(t), \quad Z_{0}(0)=z_{0} .
$$

Assume it exists and is unique in the weak sense.

Theorem 5.2. Assume $D_{n} \Rightarrow \mathbb{R}^{d}$ weakly, and $g_{n} \Rightarrow g_{0}, A_{n} \Rightarrow A_{0}, z_{n} \rightarrow z_{0}$. Then $Z_{n} \Rightarrow Z_{0}$ in $C\left([0, T], \mathbb{R}^{d}\right)$ for every $T>0$.

Proof. We modify the proof of Theorem 2.7 a bit. First, fix a compact set $\mathcal{K} \subseteq \mathbb{R}^{d}$ such that $z_{0} \in$ int $\mathcal{K}$. It suffices to show that $Z_{n}^{\mathcal{K}} \Rightarrow Z_{0}^{\mathcal{K}}$, then apply Lemma 4.1. (It is stated and proved for a non-reflected $Z_{0}$ in the same way as for the case of a reflected diffusion $Z_{0}$.) By Lemma 5.1, there exists $n_{0}$ such that $\mathcal{K} \subseteq D_{n}$ for $n>n_{0}$. So $L_{n}(t) \equiv 0$, and $Z_{n}^{\mathcal{K}} \equiv V_{n}$ (we use the notation from the proof of Theorem 2.7). The rest of the proof is reduced to Lemmata 4.3 and 4.7 .

\subsection{Convergence of domains to "almost" the whole space}

Now, assume $D_{n} \Rightarrow D_{0}=\mathbb{R}^{d} \backslash \mathcal{M}$, where $\mathcal{M} \subseteq \mathbb{R}^{d}$ is a "set of dimension" less than or equal to $d-2$. Then the limiting diffusion $Z_{0}$ (under some conditions) does not hit $\mathcal{M}$, so this is actually a non-reflected diffusion. We use the notation of the previous subsection. We again suppose that Assumptions 1, 2, 3 are satisfied, and we do not impose a condition that $Z_{n}$ does not hit non-smooth parts $\mathcal{V}_{n}$ of the boundary $\partial D_{n}$. 
Theorem 5.3. In the notation of the previous subsection, assume

$$
D_{n} \Rightarrow D_{0}=\mathbb{R}^{d} \backslash \mathcal{M}, \quad g_{n} \Rightarrow g_{0}, \quad A_{n} \Rightarrow A_{0}, \quad z_{n} \rightarrow z_{0} .
$$

Finally, assume that the diffusion $Z_{0}=\left(Z_{0}(t), t \geq 0\right)$, defined by

$$
\mathrm{d} Z_{0}(t)=g_{0}\left(Z_{0}(t)\right) \mathrm{d} t+\sigma_{0}\left(Z_{0}(t)\right) \mathrm{d} W(t), \quad Z_{0}(0)=z_{0},
$$

a.s. does not hit the set $\mathcal{M}$ :

$$
\mathbf{P}\left(\exists t \geq 0: Z_{0}(t) \in \mathcal{M}\right)=0 .
$$

Then $Z_{n} \Rightarrow Z_{0}$ in $C\left([0, T], \mathbb{R}^{d}\right)$.

Remark 4. Sufficient conditions for $Z_{0}$ not hitting $\mathcal{M}$, when $\mathcal{M}$ is a submanifold in $\mathbb{R}^{d}$ of dimension less than or equal to $d-2$, can be found in $[14,15]$.

Proof. As in the proof of Theorem 5.2, we follow the proof of Theorem 2.7. Fix any compact set $\mathcal{K} \subseteq D_{0}$. It suffices to prove that $Z_{n}^{\mathcal{K}} \Rightarrow Z_{0}^{\mathcal{K}}$. By Corollary 2.3, there exists $n_{0}$ such that for $n>n_{0}$, we have: $\mathcal{K} \subseteq D_{n}$. Now, we just need to repeat the rest of the proof of Theorem 5.2.

\section{Appendix}

\section{A.1. Proof of Lemma 2.1}

(i) Similar to the proof of Lemma 2.2 below.

(ii) Fix $\varepsilon>0$ and let us show that $\operatorname{dist}\left(x_{0}, E_{0}\right)<2 \varepsilon$. There exists $n_{1}$ such that for $n \geq n_{1}$ we have: $\left\|x_{n}-x_{0}\right\|<\varepsilon$. The set $\mathcal{K}:=\left\{x_{n} \mid n \geq 1\right\}$ is compact; therefore, $\operatorname{dist}\left(x, E_{n}\right) \rightarrow \operatorname{dist}\left(x, E_{0}\right)$ uniformly on $\mathcal{K}$, and there exists $n_{2}$ such that for $n \geq n_{2}$, we have: $\left|\operatorname{dist}\left(x, E_{n}\right)-\operatorname{dist}\left(x, E_{0}\right)\right|<\varepsilon$ for $x \in \mathcal{K}$. Take $n=n_{1} \vee n_{2}$. Then

$$
\operatorname{dist}\left(x_{0}, E_{0}\right) \leq \operatorname{dist}\left(x_{0}, E_{n}\right)+\left\|x_{n}-x_{0}\right\| \leq \operatorname{dist}\left(x_{n}, E_{n}\right)+\varepsilon+\left\|x_{n}-x_{0}\right\| \leq 2 \varepsilon .
$$

Since $\varepsilon>0$ is arbitrary, $\operatorname{dist}\left(x_{0}, E_{0}\right)=0$; therefore, $x_{0} \in \bar{E}_{0}$.

\section{A.2. Proof of Lemma 2.2}

(i) $\Rightarrow$ (iii). Assume the converse: there exists a compact subset $\mathcal{K} \subseteq \mathbb{R}^{d}$, a positive number $\varepsilon>0$ and a sequence $x_{n_{k}} \in \mathcal{K}$ such that

$$
\left|\varphi_{n_{k}}\left(x_{n_{k}}\right)-\varphi_{0}\left(x_{n_{k}}\right)\right| \geq \varepsilon
$$

By compactness, there exists a limit point $x_{0}:=\lim x_{n_{k}^{\prime}}$. There exists $k_{0}$ such that for $k \geq k_{0}$, we have: $\left\|x_{n_{k}^{\prime}}-x_{0}\right\| \leq$ $\varepsilon / 3$. But the signed distance functions $\varphi_{0}$ and $\varphi_{n_{k}^{\prime}}$ are 1-Lipschitz, see [5]. Therefore, for $k \geq k_{0}$ we get:

$$
\left|\varphi_{n_{k}^{\prime}}\left(x_{n_{k}^{\prime}}\right)-\varphi_{n_{k}^{\prime}}\left(x_{0}\right)\right| \leq \frac{\varepsilon}{3}, \quad\left|\varphi_{0}\left(x_{n_{k}^{\prime}}\right)-\varphi_{0}\left(x_{0}\right)\right| \leq \frac{\varepsilon}{3} .
$$

Thus, for $k \geq k_{0}$ we have:

$$
\left|\varphi_{n_{k}^{\prime}}\left(x_{0}\right)-\varphi_{0}\left(x_{0}\right)\right| \geq \varepsilon-\frac{\varepsilon}{3}-\frac{\varepsilon}{3}=\frac{\varepsilon}{3} .
$$

This contradicts the condition (i). 
(i) $\Leftrightarrow$ (ii). Note that $\operatorname{dist}\left(x, \bar{D}_{n}\right) \equiv\left(\varphi_{n}(x)\right)_{-}$and $\operatorname{dist}\left(x, D_{n}^{c}\right) \equiv\left(\varphi_{n}(x)\right)_{+}$. A sequence $\left(a_{n}\right)_{n \geq 1}$ of real numbers converges to $a_{0}$ if and only if $\left(a_{n}\right)_{+} \rightarrow\left(a_{0}\right)_{+}$and $\left(a_{n}\right)_{-} \rightarrow\left(a_{0}\right)_{-}$. The "only if" part follows from the fact that $x \mapsto x_{+}$and $x \mapsto x_{-}$are continuous functions; the "if" part follows from the fact that $x=x_{+}-x_{-}$. The rest is trivial.

(ii) $\Rightarrow$ (iv). Since the function $\varphi_{0}$ is 1-Lipschitz, as proved in [5], we have:

$$
\max _{\substack{x_{n}, x_{0} \in \mathcal{K} \\\left\|x_{n}-x_{0}\right\| \leq \varepsilon_{n}}}\left|\varphi_{n}\left(x_{n}\right)-\varphi_{0}\left(x_{0}\right)\right| \leq \max _{\substack{x_{n}, x_{0} \in \mathcal{K} \\\left\|x_{n}-x_{0}\right\| \leq \varepsilon_{n}}}\left|\varphi_{n}\left(x_{n}\right)-\varphi_{0}\left(x_{n}\right)\right|+\varepsilon_{n} \rightarrow 0 .
$$

(iv) $\Rightarrow$ (v). Take $\mathcal{K}=\left\{z \in \mathbb{R}^{d} \mid\|z\| \leq \max \left\|f_{0}\right\|+1\right\}$ and $\varepsilon_{n}:=\max \left\|f_{n}(t)-f_{0}(t)\right\|$ for $n=1,2, \ldots$

(v) $\Rightarrow$ (i). Take constant functions $f_{n}(t) \equiv x$ for $t \in[0, T]$ and $n=0,1,2, \ldots$

(i) $\Rightarrow$ (vi). Note that $\operatorname{dist}\left(x, \partial D_{n}\right) \equiv\left|\varphi_{n}(x)\right|$ for $x \in \mathbb{R}^{d}$ and $n=0,1,2, \ldots$ Since $\varphi_{n}(x) \rightarrow \varphi_{0}(x)$, we have: $\left|\varphi_{n}(x)\right| \rightarrow\left|\varphi_{0}(x)\right|$. Now, let us show (2). Take $x \in D_{0}$. Then $\lim \varphi_{n}(x)=\varphi_{0}(x)>0$, and so there exists $n_{x}$ such that for $n \geq n_{x}$ we get: $\varphi_{n}(x)>0$, and therefore $x \in D_{n}$. Thus, $x \in \underline{\lim } D_{n}$. We conclude that $D_{0} \subseteq \underline{\lim } D_{n}$. Similarly, we can prove that $\bar{D}_{0}^{c} \subseteq \underline{\lim }_{n \rightarrow \infty} \bar{D}_{n}^{c}$, which is equivalent to $\overline{\lim }_{n \rightarrow \infty} \bar{D}_{n} \subseteq \bar{D}_{0}$.

(vi) $\Rightarrow$ (i). We have: $\left|\varphi_{n}(x)\right| \rightarrow\left|\varphi_{0}(x)\right|$ for every $x \in \mathbb{R}^{d}$, as $n \rightarrow \infty$. Consider three cases:

Case 1: $\varphi_{0}(x)>0$, which is equivalent to $x \in D_{0}$. Using the first inclusion from (2), we get: $x \in \lim D_{n}$, and so there exists $n_{x}$ such that for $n \geq n_{x}$, we have: $x \in D_{n}$, and $\varphi_{n}(x)>0$. Therefore, $\varphi_{n}(x)=\left|\varphi_{n}(x)\right| \rightarrow\left|\varphi_{0}(x)\right|=\varphi_{0}(x)$.

Case 2: $\varphi_{0}(x)<0$, which is equivalent to $x \in \bar{D}_{0}^{c}$. Then we use the second inclusion from (2) and complete the proof similarly to Case 1 .

Case 3: $\varphi_{0}(x)=0$. Then $\left|\varphi_{n}(x)\right| \rightarrow\left|\varphi_{0}(x)\right|=0$, and so $\varphi_{n}(x) \rightarrow 0$.

(vi) $\Rightarrow$ (vii). Follows from Lemma 2.1(ii) above.

(vii) $\Rightarrow$ (vi). Fix $x \in \mathbb{R}^{d}$ and let us show that $\operatorname{dist}\left(x, \partial D_{n}\right) \rightarrow \operatorname{dist}\left(x, \partial D_{0}\right)$.

Lemma A.1. Assume (2) holds. Take $x_{0} \in \partial D_{0}$. Then there exists a sequence $\left(x_{n}\right)_{n \geq 1}$ such that $x_{n} \in \partial D_{n}$ and $x_{n} \rightarrow x_{0}$.

Proof. Assume the converse: there exists a neighborhood $U\left(x_{0}, \varepsilon\right)$ and a subsequence $\left(n_{k}\right)_{k \geq 1}$ such that for $k \geq 1$, we have: $U\left(x_{0}, \varepsilon\right) \cap \partial D_{n_{k}}=\varnothing$. Since $x_{0} \in \partial D_{0}$, there exists $y \in U\left(x_{0}, \varepsilon\right) \cap D_{0}$ and $z \in U\left(x_{0}, \varepsilon\right) \cap \bar{D}_{0}^{c}$. Then $y \in \underline{\lim } D_{n}$; that is, $y \in D_{n}$ for $n>n_{y}$; and $z \in \underline{\lim } \bar{D}_{n}^{c}$, that is, $z \in \bar{D}_{n}^{c}$ for $n>n_{z}$. Let $k_{0}$ be large enough so that for $k \geq k_{0}$, $n_{k}>n_{y} \vee n_{z}$. Then $y \in D_{n_{k}}$ and $z \in \bar{D}_{n_{k}}^{c}$ for $k \geq k_{0}$. Therefore, $[y, z] \cap \partial D_{n_{k}} \neq \varnothing$; take $w_{k} \in[y, z] \cap \partial D_{n_{k}}$. But $[y, z] \subseteq U\left(x_{0}, \varepsilon\right)$, because the open ball $U\left(x_{0}, \varepsilon\right)$ is convex. Therefore, $U\left(x_{0}, \varepsilon\right) \cap \partial D_{n_{k}} \neq \varnothing$. This contradiction completes the proof.

Let $y_{n} \in \mathbb{R}^{d}$ be the closest point on $\partial D_{n}$ to $x:\left\|x-y_{n}\right\|=\operatorname{dist}\left(x, \partial D_{n}\right)$.

Lemma A.2. The sequence $\left(y_{n}\right)_{n \geq 1}$ is bounded.

Proof. Consider three cases:

Case 1: $x \in D_{0}$. Then $x \in D_{0} \subseteq \underline{\lim }_{n \rightarrow \infty} D_{n}$. Therefore, $x \in D_{n}$ for $n \geq n_{x}$. Now, take any $y \in \bar{D}_{0}^{c}$; then $y \in \bar{D}_{0}^{c} \subseteq$ $\underline{\lim }_{n \rightarrow \infty} \bar{D}_{n}^{c}$. Therefore, $y \in D_{n}$ for $n \geq n_{y}$. Take $n \geq n_{x} \vee n_{y}$; then $x \in D_{n}$ and $y \in \bar{D}_{n}^{c}$. Therefore, $[x, y] \cap \partial D_{n} \neq \varnothing$. Take some $u_{n} \in[x, y] \cap \partial D_{n}$; then $\left\|x-y_{n}\right\| \leq \operatorname{dist}\left(x, \partial D_{n}\right) \leq\left\|x-u_{n}\right\| \leq\|x-y\|$. Thus, $\left\|y_{n}\right\| \leq\|x\|+\|x-y\|$.

Case 2: $x \in \bar{D}_{0}^{c}$. This is similar to Case 1 .

Case 3: $x \in \partial D_{0}$. Use Lemma A.1 below and find a sequence $x_{n} \in \partial D_{n}$ such that $x_{n} \rightarrow x$. Then $\left\|x-y_{n}\right\|=$ $\operatorname{dist}\left(x, \partial D_{n}\right) \leq\left\|x-x_{n}\right\| \rightarrow 0$. Therefore, $y_{n} \rightarrow x$, and $\left(y_{n}\right)_{n \geq 1}$ is bounded.

Let us show that

$$
\operatorname{dist}\left(x, \partial D_{0}\right) \leq \lim _{n \rightarrow \infty} \operatorname{dist}\left(x, \partial D_{n}\right) .
$$

Take a subsequence $\left(n_{k}\right)_{k \geq 1}$. It suffices to show that there exists a subsequence $\left(n_{k}^{\prime}\right)_{k \geq 1} \subseteq\left(n_{k}\right)_{k \geq 1}$ such that

$$
\operatorname{dist}\left(x, \partial D_{0}\right) \leq \lim _{k \rightarrow \infty} \operatorname{dist}\left(x, \partial D_{n_{k}^{\prime}}\right) .
$$


The sequence $\left(y_{n_{k}}\right)_{k \geq 1}$ is bounded by Lemma A.2. Therefore, there exists a subsequence $\left(n_{k}^{\prime}\right)_{k \geq 1} \subseteq\left(n_{k}\right)_{k \geq 1}$ such that $y_{n_{k}^{\prime}} \rightarrow \bar{y}$. By assumption (vii), $\bar{y} \in \partial D_{0}$. Therefore,

$$
\operatorname{dist}\left(x, \partial D_{0}\right) \leq\|x-\bar{y}\|=\lim _{k \rightarrow \infty}\left\|x-y_{n_{k}^{\prime}}\right\|=\lim _{k \rightarrow \infty} \operatorname{dist}\left(x, \partial D_{n_{k}^{\prime}}\right) .
$$

This proves (A.1). Now, let us show that

$$
\operatorname{dist}\left(x, \partial D_{0}\right) \geq \underset{n \rightarrow \infty}{\lim } \operatorname{dist}\left(x, \partial D_{n}\right)
$$

By Lemma A.1, there exists a sequence $\bar{y}_{n} \in \partial D_{n}$ such that $\bar{y}_{n} \rightarrow y_{0}$. Therefore, $\operatorname{dist}\left(x, \partial D_{0}\right)=\left\|x-y_{0}\right\|=$ $\lim _{n \rightarrow \infty}\left\|x-\bar{y}_{n}\right\|$. But $\left\|x-\bar{y}_{n}\right\| \leq \operatorname{dist}\left(x, \partial D_{n}\right)$. This proves (A.2).

\section{A.3. Proof of Lemma 4.11}

We need only to prove continuity of $\zeta$, the rest is done in [19, Lemma 3.2]. Let $x_{n} \rightarrow x_{0}$ in $\mathcal{K}_{0}$, and take $y_{0}$, a limit point of $\zeta\left(x_{n}\right)$. Without loss of generality assume $y_{0}=\lim _{n \rightarrow \infty} \zeta\left(x_{n}\right)$. Then $\operatorname{dist}\left(x_{n}, \partial D_{0}\right)=\left\|x_{n}-\zeta\left(x_{n}\right)\right\| \rightarrow\left\|x_{0}-y_{0}\right\|$. But the distance function is continuous. So $\left\|x_{0}-y_{0}\right\|=\operatorname{dist}\left(x_{0}, \partial D_{0}\right)$. Since the closest point on $\partial D_{0}$ to $x_{0}$ is unique, we have: $y_{0}=\zeta\left(x_{0}\right)$. The proof is complete.

\section{A.4. Proof of Corollary 2.3}

(i) The proof is trivial.

(ii) Let us prove the first statement, when $\mathcal{K} \subseteq D_{0}$; the second one is similar. From Lemma 2.2(iii) we have: $\varphi_{n}(x) \rightarrow \varphi_{0}(x)>0$ uniformly on $\mathcal{K}$, and $\varphi_{0}$ is continuous on $\mathcal{K}$. Therefore, there exists $\varepsilon>0$ such that $\varphi_{0}(x) \geq \varepsilon$ for $x \in \mathcal{K}$. By the uniform convergence, there exists $n_{0}$ such that for $n>n_{0}$ we have: $\varphi_{n}(x) \geq \varepsilon / 2>0$ for $x \in \mathcal{K}$. This completes the proof.

\section{A.5. Proof of Lemma 2.4}

Let us show the first case, when $D_{n} \uparrow D_{0}$; the second case is similar.

Case 1: $x \in D$. Then $\varphi_{0}(x)=: r>0$. There exists $n_{x}$ such that for $n \geq n_{x}$ we have: $x \in D_{n}$. Therefore, $\varphi_{n}(x)>0$ for $n \geq n_{x}$, and $\varphi_{n}(x)=\operatorname{dist}\left(x, \partial D_{n}\right)=\operatorname{dist}\left(x, D_{n}^{c}\right)$. We have: $\left(\varphi_{n}(x)\right)_{n \geq n_{x}}$ is a nondecreasing sequence, and $\varphi_{n}(x) \leq$ $\varphi_{0}(x)$ for each $n \geq n_{x}$.

Now, fix $\varepsilon>0$ and consider the closed ball $B(x, r-\varepsilon) \subseteq D$. We have: $B(x, r-\varepsilon) \subseteq \cup D_{n}$. But this ball is compact, so there exists a finite subcover $D_{n_{1}}, \ldots, D_{n_{m}}$. Take $k_{x}:=\max \left(n_{1}, \ldots, n_{m}, n_{x}\right)$. Then $B(x, r-\varepsilon) \subseteq D_{k_{x}}$. Therefore, $\varphi_{k_{x}}(x) \geq r-\varepsilon$. By monotonicity of $\left(\varphi_{n}(x)\right)_{n \geq n_{x}}$, we have: $\varphi_{n}(x) \geq r-\varepsilon=\varphi_{0}(x)-\varepsilon$ for $n \geq k_{x}$. Since $\varepsilon>0$ is arbitrary, this proves that $\varphi_{n}(x) \rightarrow \varphi_{0}(x)$ as $n \rightarrow \infty$.

Case 2: $x \notin D$. Then $\varphi_{0}(x) \leq 0$. Therefore, $\operatorname{dist}\left(x, \partial D_{0}\right)=\left|\varphi_{0}(x)\right|$. Take $y \in \partial D_{0}$ such that $\|y-x\|=\left|\varphi_{0}(x)\right|$. Fix $\varepsilon>0$; then there exists $z \in D$ such that $\|z-y\| \leq \varepsilon$. Therefore, $\|z-x\| \leq\|z-y\|+\|y-x\| \leq\left|\varphi_{0}(x)\right|+\varepsilon$. Because $D=\bigcap D_{n}$, there exists $n_{0}$ such that $z \in D_{n}$ for $n \geq n_{0}$. Therefore, $\operatorname{dist}\left(x, D_{n}\right) \leq\left|\varphi_{0}(x)\right|+\varepsilon$. But $x \notin D_{n}$ for all $n \geq 1$; therefore, $-\operatorname{dist}\left(x, D_{n}\right)=\varphi_{n}(x)$. But $\operatorname{dist}\left(x, D_{n}\right) \leq\|x-z\| \leq\left|\varphi_{0}(x)\right|+\varepsilon$. Therefore,

$$
\varphi_{n}(x) \geq-\left|\varphi_{0}(x)\right|-\varepsilon=\varphi_{0}(x)-\varepsilon \quad \text { for } n \geq n_{0} .
$$

But $D_{n} \uparrow D_{0}$, and $x \notin D_{0}$. Therefore, $\left(\operatorname{dist}\left(x, D_{n}\right)\right)_{n \geq 1}$ is nonincreasing, and so $\left(\varphi_{n}(x)=-\operatorname{dist}\left(x, D_{n}\right)\right)_{n \geq 1}$ is nondecreasing. Also, $\operatorname{dist}\left(x, D_{n}\right) \geq \operatorname{dist}\left(x, D_{0}\right)$, and so

$$
\varphi_{n}(x)=-\operatorname{dist}\left(x, D_{n}\right) \leq-\operatorname{dist}\left(x, D_{0}\right)=\varphi_{0}(x) .
$$

Therefore, $\left(\varphi_{n}(x)\right)_{n \geq 1}$ is a nonincreasing sequence, bounded below by $\varphi_{0}(x)$. Together with (A.3), this gives $\varphi_{n}(x) \rightarrow$ $\varphi_{0}(x)$ as $n \rightarrow \infty$. 


\section{A.6. Proof of Lemma 2.5}

(i) The fact that $D_{n} \Rightarrow D_{0}$ implies Wijsman convergence follows from Lemma 2.2(ii). Now, let us give a counterexample which shows that weak convergence does not coincide with Wijsman convergence. Take the following sequence of domains in $\mathbb{R}^{2}$ :

$$
D_{n}:=\operatorname{int}\left[\left(\mathbb{R} \times \mathbb{R}_{+}\right) \backslash\left(\left[2^{-n-1}, 2^{-n}\right] \times[0,1]\right)\right], \quad n=1,2, \ldots,
$$

and the limiting domain $D_{0}=\mathbb{R} \times(0, \infty)$. Then $D_{n} \rightarrow D_{0}$ in Wijsman topology, but not in the weak sense. Indeed, for $x_{0}=(0,1)^{\prime}$ we have: $\varphi_{n}\left(x_{0}\right) \leq 2^{-n-1}$, because the distance from $x_{0}$ to the boundary $\partial D_{n}$ is less than or equal to the distance to the point $\left(2^{-n-1}, 1\right)^{\prime}$ on the boundary. But $\varphi_{0}\left(x_{0}\right)=1$, because the distance from $x_{0}$ to the boundary $\partial D_{0}$ (which is the $x_{1}$-axis) is equal to 1 . This contradicts that $\varphi_{n}\left(x_{0}\right) \rightarrow \varphi_{0}\left(x_{0}\right)$.

(ii) Now, Hausdorff convergence implies weak convergence: if $D_{n} \rightarrow D_{0}$ in Hausdorff sense, then $D_{n} \rightarrow D_{0}$ in Wijsman sense, but also $D_{n}^{c} \rightarrow D_{0}^{c}$ in Hausdorff sense, so $D_{n}^{c} \rightarrow D_{0}^{c}$ in Wijsman sense; use Lemma 2.2(ii) and complete the proof.

But weak convergence does not imply Hausdorff convergence. Indeed, let $d=2$ and $D_{0}:=\mathbb{R}_{2}^{+}$, and $D_{n}$ be the result of rotation of $D_{0}$ counterclockwise by angle $\alpha_{n}$ around the origin, where $\alpha_{n} \rightarrow 0$. Then $D_{n} \Rightarrow D_{0}$ (this is a particular case of Theorem 3.2 below), but not $D_{n} \rightarrow D_{0}$ in Hausdorff sense.

(iii) If $\varphi_{n}(x) \rightarrow \varphi_{0}(x)$ uniformly on $\mathbb{R}^{d}$, then $\left(\varphi_{n}(x)\right)_{-}=\operatorname{dist}\left(x, D_{n}\right) \rightarrow \operatorname{dist}\left(x, D_{0}\right)=\left(\varphi_{0}(x)\right)_{-}$uniformly on $\mathbb{R}^{d}$. Therefore, $D_{n} \rightarrow D_{0}$ in Hausdorff topology. Conversely, if $D_{n} \rightarrow D_{0}$ in Hausdorff topology, then $D_{n}^{c} \rightarrow D_{0}^{c}$ in Hausdorff topology, and $\operatorname{dist}\left(x, D_{n}\right) \equiv\left(\varphi_{n}(x)\right)_{-} \rightarrow \operatorname{dist}\left(x, D_{0}\right) \equiv\left(\varphi_{0}(x)\right)_{-}, \operatorname{dist}\left(x, D_{n}^{c}\right) \equiv\left(\varphi_{n}(x)\right)_{+} \rightarrow \operatorname{dist}\left(x, D_{0}^{c}\right) \equiv$ $\left(\varphi_{0}(x)\right)_{+}$, uniformly on $\mathbb{R}^{d}$. Adding these convergence relations and noting that $a \equiv a_{+}+a_{-}$for $a \in \mathbb{R}^{d}$, we complete the proof.

\section{A.7. Proof of Lemma 2.6}

(i) $\Rightarrow$ (ii). Without loss of generality, assume $n_{k}=k$. Take a sequence $\left(x_{n}\right)_{n \geq 0}$ of functions, as described in Lemma 2.6. Assume that $f_{n}\left(x_{n}\right)$ does not converge to $f_{0}\left(x_{0}\right)$ uniformly. Then there exists $\varepsilon>0$, a subsequence $\left(m_{k}\right)_{k \geq 1}$, and a sequence $\left(t_{m_{k}}\right)_{k \geq 1}$ in $[0, T]$ such that

$$
\left|f_{m_{k}}\left(x_{m_{k}}\left(t_{m_{k}}\right)\right)-f_{0}\left(x_{0}\left(t_{m_{k}}\right)\right)\right| \geq \varepsilon .
$$

We can extract a convergent subsequence $t_{m_{k}^{\prime}} \rightarrow t_{0} \in[0, T]$. Then

$$
x_{m_{k}^{\prime}}\left(t_{m_{k}^{\prime}}\right) \rightarrow x_{0}\left(t_{0}\right), \quad \text { and } \quad x_{0}\left(t_{m_{k}^{\prime}}\right) \rightarrow x_{0}\left(t_{0}\right) .
$$

Therefore, since $f_{n} \rightarrow f_{0}$ locally uniformly and $f_{0}$ is continuous on $E_{0}$,

$$
f_{m_{k}^{\prime}}\left(x_{m_{k}}\left(t_{m_{k}}\right)\right) \rightarrow f_{0}\left(x_{0}\left(t_{0}\right)\right), \quad \text { and } \quad f_{0}\left(x_{0}\left(t_{m_{k}}\right)\right) \rightarrow f_{0}\left(x_{0}\left(t_{0}\right)\right) .
$$

This contradicts (A.4).

(ii) $\Rightarrow$ (i). Take $x_{n_{k}}(t) \equiv z_{n_{k}}$ and $x_{0}(t) \equiv z_{0}$.

\section{A.8. Proof of Lemma 2.8}

Assume the condition (b) holds. Take a sequence $x_{n_{k}} \in \mathcal{V}_{n_{k}}$ such that $x_{n_{k}} \rightarrow x_{0}$. Since the set $\mathcal{K}:=\overline{\left\{x_{n_{k}} \mid k=1,2, \ldots\right\}}$ is compact, from condition (b) we have: $\operatorname{dist}\left(x_{n_{k}}, \mathcal{V}_{0}\right) \rightarrow 0$. The function $\operatorname{dist}\left(\cdot, \mathcal{V}_{0}\right)$ is continuous. Therefore, $\operatorname{dist}\left(x_{0}, \mathcal{V}_{0}\right)=0$, which means $x_{0} \in \mathcal{V}_{0}$ (because the set $\mathcal{V}_{0}$ is closed). Conversely, assume that for every sequence $\left(x_{n_{k}}\right)_{k \geq 1}$ such that $x_{n_{k}} \in \mathcal{V}_{n_{k}}$ and $x_{n_{k}} \rightarrow x_{0}$ we have: $x_{0} \in \mathcal{V}_{0}$. Take a compact set $\mathcal{K} \subseteq \mathbb{R}^{d}$. Let us show (6). Assume the converse: there exists $\varepsilon>0$ and a subsequence $\left(n_{k}\right)_{k \geq 1}$ such that

$$
\max _{x \in \mathcal{V}_{n_{k}} \cap \mathcal{K}} \operatorname{dist}\left(x, \mathcal{V}_{0}\right)>\varepsilon
$$


Then there exists $x_{n_{k}} \in \mathcal{V}_{n_{k}} \cap \mathcal{K}$ such that $\operatorname{dist}\left(x_{n_{k}}, \mathcal{V}_{0}\right)>\varepsilon$. Now, the sequence $\left(x_{n_{k}}\right)_{k \geq 1}$ is bounded, so there exists a limit point $\bar{x}:=\lim x_{n_{k}^{\prime}}$. Therefore, $\bar{x} \in \mathcal{V}_{0}$ by our assumption. And

$$
\operatorname{dist}\left(\bar{x}, \mathcal{V}_{0}\right)=\lim _{k \rightarrow \infty} \operatorname{dist}\left(x_{n_{k}^{\prime}}, \mathcal{V}_{0}\right) \geq \varepsilon
$$

This contradiction completes the proof.

\section{A.9. Proof of Lemma 5.1}

Let us show the "only if" part. Take a compact set $\mathcal{K} \subseteq \mathbb{R}^{d}$ and assume that there exists a subsequence $\left(n_{k}\right)_{k \geq 1}$ such that for some $x_{n_{k}} \in \mathcal{K}$, we have: $x_{n_{k}} \notin D_{n_{k}}$. Extract a convergent subsequence: $x_{n_{k}^{\prime}} \rightarrow y \in \mathcal{K}$. We claim that

$$
\varlimsup_{k \rightarrow \infty} \varphi_{n_{k}^{\prime}}(y) \leq 0 .
$$

Indeed, if $y \notin D_{n_{k}^{\prime}}$, then $\varphi_{n_{k}^{\prime}}(y) \leq 0$. If $y \in D_{n_{k}^{\prime}}$, then $\varphi_{n_{k}^{\prime}}(y)=\operatorname{dist}\left(y, \partial D_{n_{k}^{\prime}}\right)=\operatorname{dist}\left(y, D_{n_{k}^{\prime}}^{c}\right) \leq\left\|y-x_{n_{k}^{\prime}}\right\| \rightarrow 0$. This proves the claim (A.5). But this contradicts the assumption that $\varphi_{n}(y) \rightarrow \infty$.

Now, let us show the "if" part. Take $x \in \mathbb{R}^{d}$ and let $\mathcal{K}:=\overline{U(x, N)}$ for large $N$. By assumption, there exists $n_{0}$ such that for $n>n_{0}$ we have: $\mathcal{K} \subseteq D_{n}$. So $x \in D_{n}$, and $\varphi_{n}(x)=\operatorname{dist}\left(x, \partial D_{n}\right) \geq N$. Since $N$ is arbitrarily large, this completes the proof.

\section{Acknowledgements}

The author would like to thank KRZYSZTOF BURDZY, IOANNIS KARATZAS, RUTH WiLliams, for help and useful discussion. The author would also like to express his gratitude to two anonymous referees, who pointed out many mistakes and misprints. This research was partially supported by NSF grants DMS 1007563, DMS 1308340, DMS 1405210, DMS 1409434.

\section{References}

[1] G. Beer. Wijsman convergence: A survey. Set-Valued Var. Anal. 2 (1-2) (1994) 77-94. MR1285822

[2] K. Burdzy and Z.-Q. Chen. Weak convergence of reflected Brownian motions. Electron. J. Probab. 3 (4) (1998) 29-33. MR1625707

[3] K. Burdzy and D. Marshall Hitting a boundary point with reflected Brownian motion. In Séminaire de Probabilités, XXVI 81-94. Lecture Notes in Math. 1526. Springer, 1992. MR1231985

[4] J. G. Dai and R. J. Williams. Existence and uniqueness of semimartingale reflecting Brownian motions in convex polyhedra. Theory Probab. Appl. 40 (1) (1995) 3-53. MR1346729

[5] M. C. Delfour and J.-P. Zolesio. Shapre analysis via oriented distance functions. J. Funct. Anal. 123 (1) (1994) 129-201. MR1279299

[6] J. M. Harrison and I. M. Reiman. Reflected Brownian motion on an orthant. Ann. Probab. 9 (2) (1981) 302-308. MR0606992

[7] R. A. Horn and C. R. Johnson. Matrix Analysis. Cambridge University Press, 1990. MR1084815

[8] S. Hottovy. The Smoluchowski-Kramers approximation for stochastic differential equations with arbitrary state-dependent friction. Ph.D. Thesis, 2013. MR3153357

[9] N. Ikeda and S. Watanabe. Stochastic Differential Equations and Diffusion Processes. North-Holland, 1989. MR0637061

[10] W. Kang and K. Ramanan. On the submartingale problem for reflected diffusions in domains with piecewise smooth boundaries, 2014. Available at arXiv:1412:0729. MR3601653

[11] W. Kang and R. J. Williams. An invariance principle for semimartingale reflecting Brownian motions in domains with piecewise smooth boundaries. Ann. Appl. Probab. 17 (2) (2007) 741-779. MR2308342

[12] T. G. Kurtz and P. Protter. Weak limit theorems for stochastic integrals and stochastic differential equations. Ann. Probab. 19 (3) (1991) 1035-1070. MR1112406

[13] J. R. Munkres. Topology: A First Course. Prentice-Hall, 1975. MR0464128

[14] S. Ramasubramanian. Recurrence of projections of diffusions. Sankhya A 45 (1) (1983) 20-31. MR0749350

[15] S. Ramasubramanian. Hitting of submanifolds by diffusions. Probab. Theory Related Fields 78 (1) (1988) 149-163. MR0940875

[16] I. M. Reiman and R. J. Williams. A boundary property of semimartingale reflecting Brownian motions. Probab. Theory Related Fields 77 (1) (1988) 87-97. MR0921820

[17] A. Sarantsev. Triple and simultaneous collisions of competing Brownian particles. Electron. J. Probab. 20 (1) (2015) 1-29. MR3325099

[18] A. Sarantsev. Infinite systems of competing Brownian particles, 2016. Available at arXiv:1403.4229. MR3438889

[19] A. Sarantsev. Penalty method for obliquely reflected diffusions, 2016. Available at arXiv:1509.01777. 
[20] L. M. Taylor and R. J. Williams. Existence and uniqueness of semimartingale reflecting Brownian motions in an orthant. Probab. Theory Related Fields 75 (4) (1993) 459-485. MR1231926

[21] R. A. Wijsman. Convergence of sequences of convex sets, cones and functions II. Trans. Amer. Math. Soc. 123 (1966) 32-45. MR0196599

[22] R. J. Williams. Reflected Brownian motion with skew-symmetric data in a polyhedral domain. Probab. Theory Related Fields 75 (4) (1987) 459-485. MR0894900

[23] R. J. Williams. Semimartingale reflecting Brownian motions in the orthant. In Stochastic Networks 125-137. IMA Vol. Math. Appl. 71. Springer, 1995. MR1381009

[24] R. J. Williams. An invariance principle for semimartingale reflecting Brownian motions in an orthant. Queueing Syst. 30 (1-2) (1998) 5-25. MR1663755 